\title{
5. SEDIMENTOLOGY OF THE COLLISION ZONE BETWEEN THE NORTH D'ENTRECASTEAUX RIDGE AND NEW HEBRIDES ISLAND ARC (SITES 827, 828, AND 829) ${ }^{1}$
}

\author{
R. Pamela Reid, ${ }^{2}$ Steven N. Carey, ${ }^{3}$ T. Scott Staerker ${ }^{4}$ and Maria Ask $^{5}$
}

\begin{abstract}
Depositional processes in the collision zone between the North d'Entrecasteaux Ridge (NDR) and the New Hebrides Island Arc were investigated in post-cruise analyses of sediment and nannofossils from Sites 827, 828, and 829. Reexamination of cores and analysis of grain size, turbidite frequency, carbonate content, and clay mineralogy, together with new nannofossil age determinations, were used to revise shipboard lithostratigraphies and interpret the sedimentologic history recorded at each site.

The results of our analyses show that sedimentation on the inner trench slope and the impinging NDR has been significantly affected by the collision process. Uplift associated with subduction of the NDR beneath the New Hebrides Island Arc resulted in the emergence of Espiritu Santo Island and created a robust source of epiclastic sediment for the forearc area. Distribution of this material into deeper water has been modulated by Pleistocene sea level changes, which have influenced the frequency and extent of turbidity currents. Sedimentation at individual sites reflects the interplay between sediment source availability, sea level changes, and tectonic displacements, which have translated local depositional environments both horizontally and vertically. The study also provides evidence that ridge sediment and trench fill deposits have been accreted onto the forearc slope and that thrust faulting of subducting ridge sediment has occurred preferentially in kaolinite-rich horizons.
\end{abstract}

\section{INTRODUCTION}

One of the principal objectives of Leg 134 was to investigate the effect of the subduction of two different structural elements, the North d'Entrecasteaux Ridge (NDR) and Bougainville Guyot, on the forearc area of the New Hebrides Island Arc. Together these elements constitute the d'Entrecasteaux Zone, which currently impinges on the central part of the New Hebrides Island Arc. In the vicinity of the collision zone, the NDR consists of a nearly continuous ridge from which igneous rocks with mid-ocean ridge basalt (MORB) and island arc tholeiite (IAT) affinities have been dredged (Collot and Fisher, 1992; Maillet et al., 1983). Bougainville Guyot to the south is part of the South d'Entrecasteaux Chain (SDC), a linear chain of seamounts and guyots (Collot et al., 1985). Dredge rocks from the SDC are of andesitic composition, indicating a strong island arc affinity (Collot et al., 1992).

Significant differences exist in the response of the inner slope trench to the underthrusting of the NDR and SDC. The NDR can be traced seismically for $15 \mathrm{~km}$ eastward beneath the toe of the deformation front. Its presence has resulted in the uplift of a broad area of the inner and upper trench slope by perhaps as much as $1500-2000$ m (Collot and Fisher, 1992). The uplifted area just west of Espiritu Santo Island is known as Wousi Bank (Fig. 1). In contrast to the uplifted slope in the NDR collision zone, the inner trench slope east of Bougainville Guyot is conspicuously indented, and shortening has occurred on a series of east-dipping thrust faults (Collot and Fisher, 1992). The trace of the subducting guyot can only be recognized for a distance of 1-2 km beneath the subduction zone.

\footnotetext{
1 Greene, H.G., Collot, J.-Y., Stokking, L.B., et al., 1994. Proc. ODP, Sci. Results, 134: College Station, TX (Ocean Drilling Program)

${ }^{2}$ Rosenstiel School of Marine and Atmospheric Science, University of Miami, Miami FL 33149, U.S.A.

${ }^{3}$ University of Rhode Island, Graduate School of Oceanography, Narragansett, RI 02882, U.S.A.

${ }^{4}$ Department of Geology, Florida State University, Tallahassee, FL 32306, U.S.A (Present address: EA Engineering, Science, and Technology, Carrollton, TX 75006, U.S.A.)

${ }^{5}$ Division of Rock Mechanics, Lulea University of Technology, S-951 87 Lulea, Sweden. (Present address: Engineering Geology, Royal Institute of Technology, S-100 44 Stockholm, Sweden.)
}

Sites 827,828 , and 829 were drilled in the area of the NDR collision zone to study the nature of the ridge-arc interaction. Sites 827 and 829 are located on the inner arc slope, whereas Site 828 is on the NDR just west of the deformation front. Initial examination of the cores from these sites onboard the Joides Resolution suggests that the forearc slope is comprised both of sediment derived from the arc and deposited in situ, and accreted sediment, some of which is extensively sheared and fractured (Collot, Greene, Stokking, et al., 1992). In this paper, we report results of additional sedimentologic analysis of Sites 827,828 , and 829 . The objectives of our work were to determine processes of in-situ deposition on the forearc slope, the influence of arc-ridge collision on the evolution of sedimentary processes, and the lithology, age, and origin of accreted sediment. We were also interested in assessing the timing of accretion and evaluating the role of clay minerals within thrust zones. The study involved re-examination of cores from Sites 827,828 , and 829 , analysis of sediment grain size, turbidite frequency, carbonate content and clay mineralogy, as well as X-radiography. Results of the sedimentological analysis were used in conjunction with new nannofossil data for the three sites (Staerker, this volume) to revise shipboard lithostratigraphies.

As a result of NDR underthrusting of the forearc area, Sites 827 , 828 , and 829 have experienced significant vertical and lateral movements that have translated them into a variety of sedimentary environments. At the same time, the collision process has also influenced the supply of sediment from the subaerial arc. The lithostratigraphy of the sites thus reflects variations in locus of deposition and sediment source that result from complicated tectonic interactions in the forearc area. Superimposed on the tectonic control of sedimentation is a modulating effect caused by sea-level changes during the Pleistocene, which is reflected in variations in the frequency of turbidite deposition in the forearc. Profound changes in sediment accumulation rate, depositional style, and sediment provenance are to be expected in such environments where the subduction of structural elements results in significant deformation of the forearc.

\section{METHODS}

Cores from Sites 827,828 , and 829 were redescribed and sampled at the Ocean Drilling (ODP) Core Repository, Texas A\&M University, College Station, Tex. Particular emphasis was placed on sampling 


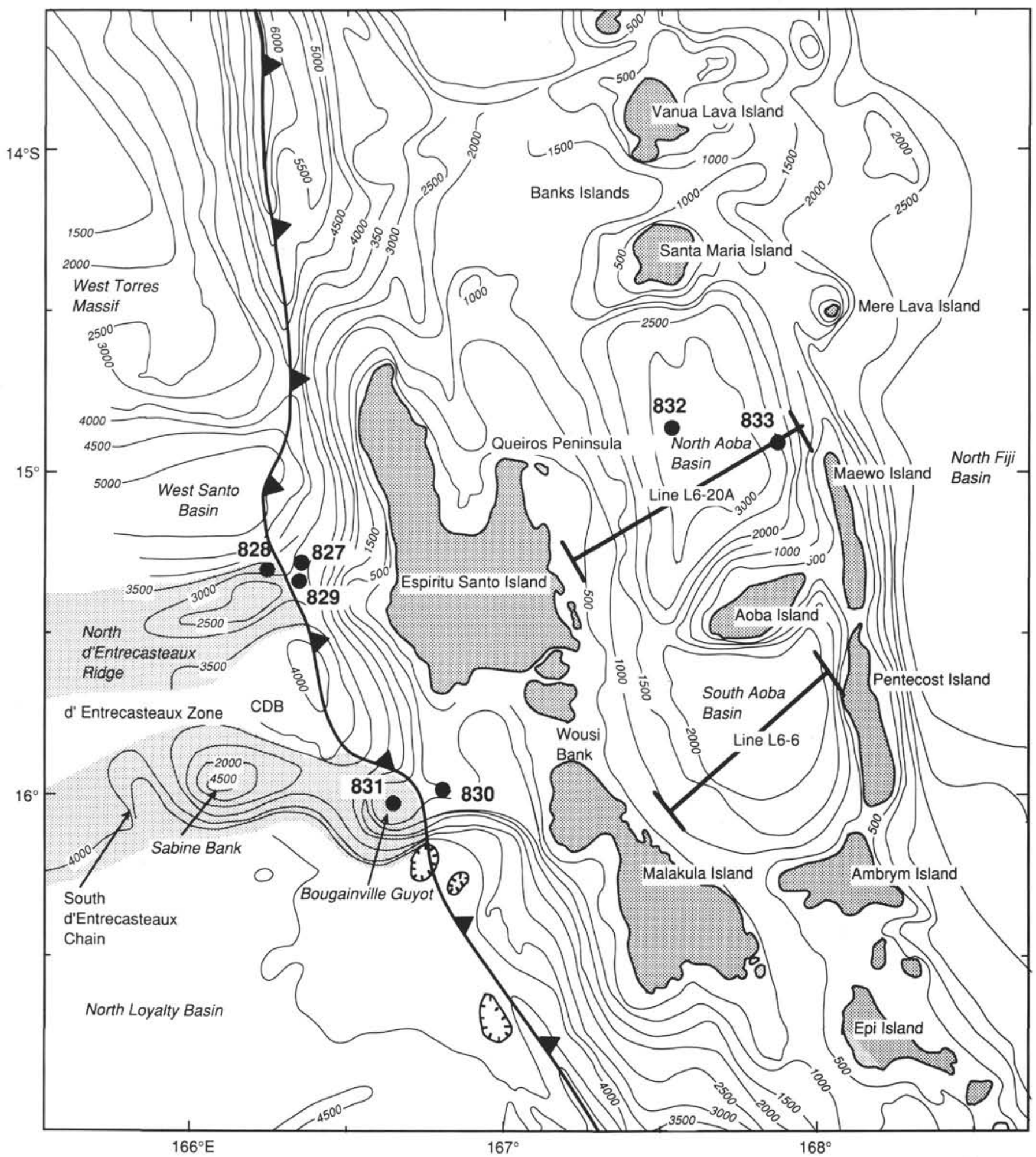

Figure 1. Map showing the locations of Sites 828, 827, and 829 in the collision zone between the North d'Entrecasteaux Ridge and the New Hebrides Island Arc.

individual clasts from intervals of conglomerate or breccia for paleontological age determination.

Grain-size analysis was carried out on 50 samples of volcanic silt layers and intervening sediment. The samples were freeze dried, weighed, and wet sieved at $62 \mu \mathrm{m}$. The amount of sand-sized sediment was determined by weighing the material retained on the sieve. The size distribution of the silt fraction $(4-62 \mu \mathrm{m})$ was analyzed with an
Elzone 180XY particle size analyzer at the Graduate School of Oceanography, University of Rhode Island. As most of the $<62 \mu \mathrm{m}$ fractions settled relatively quickly, the amount of clay-sized sediment $(<4 \mu \mathrm{m})$ was considered minor $(<10 \%)$ and was not determined quantitatively. Histograms and cumulative curves were plotted for each sample, and median grain size, sorting, and skewness were calculated. Because the sand fraction was not subdivided and most of 
the cumulative curves were lacking values for $\phi 5$ (i.e., the particle size on the cumulative curve expressed in $\phi$ units corresponding to a total cumulative weight per cent of 5), we used quartile measures of sorting and skewness instead of inclusive parameters. In cases where the cumulative curves lacked values for $\phi 25$, straight lines were drawn from the end of the curve to $\phi 2$, assuming that most grains are smaller than $250 \mu \mathrm{m}$. Statistical parameters were defined as follows: median grain size $\phi 50$; sorting (phi quartile deviation $)=(\phi 75-\phi 25) / 2$; and skewness (quartile skewness) $=(\phi 25+\phi 75)-(2 \phi 50 / 2)$.

The frequency and thickness of two types of layers (sandy layers rich in crystal and lithic grains and tephra layers rich in volcanic glass, scoria and pumice fragments) were counted from core photographs using an Olympus stereo microscope with a Cue-2 image analysis system. Images of cores were acquired with a SONY CCD camera attached to the microscope and then processed using gray level smoothing to enhance the contrast of sediment texture and allow for easier layer identification. Only layers that extended more than halfway across the core were counted. Thickness of both the sandy and ashy layers and the intervening silt intervals were measured directly from the CRT image.

The carbonate content of 232 samples was determined using a modified version of the gasometric apparatus described by Jones and Kaiteris (1983). The apparatus uses a sensitive differential pressure transducer to measure the pressure increase from $\mathrm{CO}_{2}$ evolved from the acidification of the sediment sample; it gives a routine analytical precision of $\pm 1 \%$.

Clay minerals in four samples from Sites 828 and 829 were analyzed using X-ray diffraction. Samples were disaggregated and decarbonated in a sodium acetic acid buffer at pH 5 for $12 \mathrm{hrs}$ on a hot plate at low temperature. Separation of grains smaller than $2 \mu \mathrm{m}$ was accomplished through centrifugation. The Millipore filter transfer method was used to prepare an oriented clay mineral aggregate saturated with a $1 \mathrm{M}$ solution of magnesium chloride (Moore and Reynolds, 1989). Samples were X-rayed between $2^{\circ}$ and $15^{\circ}(2 \theta)$ at a scanning speed $1 \% \mathrm{~min}$ before and after ethylene glycol treatment. Semiquantitative evaluation of the relative proportions of clay minerals was made by drawing a background line under the $17 \AA$ (glycolated smectite), $10 \AA$ (illite), and $7 \AA$ (kaolinite + chlorite) peaks and measuring peak areas with a planimeter; peak areas were weighted as follows: $1 \times$ smectite, $2 \times$ kaolinite + chlorite, and $4 \times$ illite, following Biscayne (1965).

The archive halves of five sections (Sections 134-827A-3H-3, $-827 \mathrm{~A}-15 \mathrm{X}-4,-828 \mathrm{~A}-6 \mathrm{H}-6,-829 \mathrm{~A}-4 \mathrm{R}-2$, and $-829 \mathrm{~A}-12 \mathrm{R}-2$ ) were $\mathrm{X}$-rayed to identify possible layering in sediment that appeared to be homogeneous.

Ages reported in this paper are based on post-cruise analysis of nannofossils; the complete data set is reported by Staerker (this volume). A detailed stratigraphy for the Pleistocene section of Site 828 was determined using pelagic foraminifers (see Martinez, this volume), but shipboard foraminiferal stratigraphies for Sites 827 and 829 were not refined.

\section{RESULTS}

Results of post-cruise sedimentologic analyses and nannofossil age determinations are incorporated in the following revised descriptions of the lithostratigraphic units defined by Collot, Greene, Stokking, et al. (1992). Site 828, which serves as the reference site for sediment deposited on the ridge, is discussed first.

\section{Site 828}

Site 828 is located on the northern flank of the NDR about $2 \mathrm{~km}$ west of the trace of the subduction zone and $40 \mathrm{~km}$ from the western shore of Espiritu Santo Island. It is situated on a flat, terrace-like feature that projects out from an otherwise steeply dipping slope at a water depth of $3086.7 \mathrm{~m}$. The sequence recovered at Site 828 consists of $91 \mathrm{~m}$ of upper Eocene to upper Pleistocene marine sediment overlying volcanic breccia. The sediment was subdivided onboard ship into 4 lithostratigraphic units (Units I-IV). Additions and modifications to the shipboard descriptions of these units are presented below; a revised lithostratigraphic column for the Site 828 is shown in Figure 2.

Unit I (0-61.9 meters below sea floor [mbsf]; Sections 134-828A$1 \mathrm{H}, 0 \mathrm{~cm}$, to $-8 \mathrm{H}-1)$ consists of upper Pleistocene $(\mathrm{CN} 15)$ volcanic silt that grades downward to middle or lower Pleistocene (CN14)
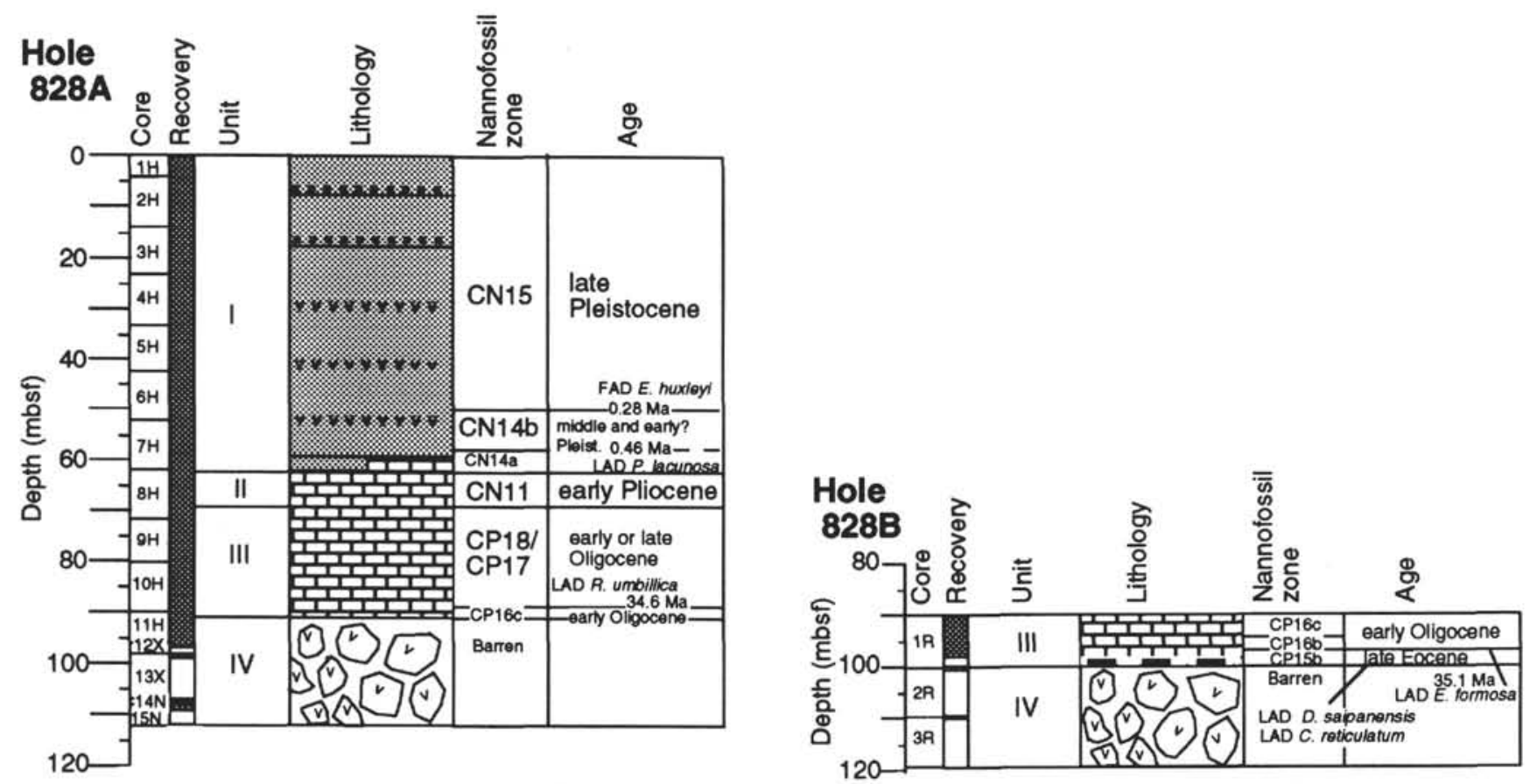

Figure 2. Revised lithostratigraphy for Site 828 . 
calcareous silt, mixed sediment, and ooze. The base of Unit I was changed from $58.7 \mathrm{mbsf}$, as defined aboard ship, to $61.9 \mathrm{mbsf}$ to include sediment in the interval from Sections 134-828A-7H-5, 83 $\mathrm{cm}$, to $-8 \mathrm{H}-1,48 \mathrm{~cm}$, which was formerly described as foraminiferal ooze and placed in Subunit IIA. As most of this sediment contains less than $60 \% \mathrm{CaCO}_{3}$ (Table 1), it is redescribed as mixed sediment with minor ooze and is included within Unit I because there is a general increase in $\mathrm{CaCO}_{3}$ throughout the lower $10 \mathrm{~m}$ of the unit.

Two types of layers, crystal/lithic-rich and volcanic glass-rich, are found throughout Unit I (Fig. 3). The crystal/lithic layers are composed primarily of sand and silt-sized volcanic minerals, such as plagioclase and pyroxenes, volcanic rock fragments, and up to $10 \%$ glass shards, pumice, and scoria fragments. They occur as normally graded layers with sharp bases and are interpreted as the deposits from dilute turbidity currents composed of epiclastic material. Volcanic glass-rich layers are composed primarily of glass, pumice, and scoria fragments. These layers are interpreted as deposits from the settling of volcanic ash through the water column following explosive volcanic eruptions in the New Hebrides Island Arc. The crystal/lithic layers are confined mainly to the upper $20 \mathrm{~m}$ of the unit, where they occur with a frequency of 4 to 17 layers per section (i.e., per $1.5 \mathrm{~m}$ of core); the average frequency is 9 layers per section. The mean thickness of the crystal/lithic layers ranges from 0.3 to $1.2 \mathrm{~cm}$ (average $=$ $0.8 \mathrm{~cm}$ ); the maximum thickness of an individual layer is $4 \mathrm{~cm}$. Most of these layers are normally graded with sharply defined bases. The glass-rich layers occur below a depth of $20 \mathrm{mbsf}$ and the bases of these layers are typically less sharply defined than the sandy layers, often having a smeared appearance. The frequency of the glass-rich layers ranges from 0 to 8 layers/section (average $=1 /$ section), mean thickness ranges from 0.3 to $2.5 \mathrm{~cm}$ (average $=1.2 \mathrm{~cm}$ ), and the maximum thickness of an individual layer is $2.7 \mathrm{~cm}$. X-radiographs show no evidence of layering within the silt intervals between the crystal/lithic and glass-rich layers.

Sediment in the upper $20 \mathrm{~m}$ of Unit I is generally coarser than that in the lower $40 \mathrm{~m}$ of the unit (Fig. 4). The silt in the upper $20 \mathrm{~m}$ has a median grain size of $38 \mu \mathrm{m}$. This silt is well sorted, and the grain-size distribution is finely skewed; this sediment contains less than $10 \%$ sand-size material. Median grain size of the crystal/lithic layers in the upper $20 \mathrm{~m}$ of Unit I ranges from 27 to $62 \mu \mathrm{m}$. The sandy sediment is moderately to poorly sorted, finely to coarsely skewed, and contains $15 \%$ to $50 \%$ sand-size material. In the lower $40 \mathrm{~m}$ of Unit I, median grain size of the silt decreases to 16 to $20 \mu \mathrm{m}$, sorting is moderate, and the grain-size distribution is near symmetrical to coarsely skewed. The glass-rich layers in this lower $40 \mathrm{~m}$ are similar to the silt in size, sorting, and skewness.

An unconformity marked by an abrupt color change separates gray mixed sediment at the base of Unit I from yellowish-brown, soupy foraminiferal ooze in Unit II (61.9-69.3 mbsf; Sections 134-828A$8 \mathrm{H}-1,48 \mathrm{~cm}$, to $-8 \mathrm{H}-6,42 \mathrm{~cm})$. The ooze of Unit II is early Pliocene (CN11) in age.

A second unconformity separates Units II and III. Unit III (69.3$90.8 \mathrm{mbsf}$ in Hole $828 \mathrm{~A}$, Sections $134-828 \mathrm{~A}-8 \mathrm{H}-6,42 \mathrm{~cm}$, to $-11 \mathrm{H}-1$, $92 \mathrm{~cm}$, and $90.0-100.0 \mathrm{mbsf}$ in Hole 828B, Sections 134-828B-1R-1, $0 \mathrm{~cm}$, to $-2 \mathrm{R}-1,0 \mathrm{~cm})$ consists mainly of lower Oligocene $(\mathrm{CP} 16 \mathrm{~b}-\mathrm{c})$ and lower or upper Oligocene (CP17/18) firm nannofossil ooze with sporadically distributed pumice fragments up to $3 \mathrm{~cm}$ in diameter and, at the top of the unit, manganese nodules. Analyses of the mineralogy and chemistry of the manganese nodules by Dr. J. Hein of the United States Geological Survey (unpublished data) suggest they formed by hydrogenetic $\mathrm{Fe}-\mathrm{Mn}$ precipitation (i.e., they were precipitated from cold ambient seawater), with little evidence of hydrothermal input. In addition to the Oligocene sediment, upper Eocene (CP15b) ooze interlaminated with brown kaolinitic clay occurs at the base of Unit III in Hole 828B. Sediment in Unit III is described as "firm ooze," rather than "chalk" as in the shipboard reports, because the sediment is not brittle and deforms with finger pressure.
Unit IV (90.8-111.4 mbsf in Hole 828A, Sections 134-828A-11H$1,92 \mathrm{~cm}$, to $-15 \mathrm{~N}-2,185 \mathrm{~cm}$, and $100.0-119.4 \mathrm{mbsf}$ in Hole $828 \mathrm{~B}$, Sections 134-828B-2R-1, 0 cm, to -3R-CC, $12 \mathrm{~cm}$ ) consists of immature volcanic breccia, brecciated lava flow deposits, and brecciated aphyric basalt and diabase. The unit is barren of nannofossils, but middle Eocene pelagic foraminifers were found in Section 134-829A11H-CC (Collot, Greene, Stokking, et al., 1992). Smectite occurs in the fractures of some of the volcanic rock fragments (Coltorti et al., this volume).

\section{Sedimentologic History}

The igneous rocks recovered at the base of Site 828 are interpreted as the eroded and weathered detritus of a volcanic basement complex that includes rocks with a MORB affinity and rocks that are transitional in composition between MORB and IAT (Collot, Greene, Stokking, et al., 1992; Coltorti et al., this volume). The sedimentary sequence above the volcanic complex records a transition from pelagic sedimentation in late Eocene, Oligocene, and early Pliocene times, to hemipelagic sedimentation dominated by volcaniclastic input during middle and late Pleistocene time. (The terms pelagic and hemipelagic are used in the sense of Pickering et al., [1989, pp. 34-35].) Pelagic grains initially enter the hydrosphere beyond the shelf-slope break, or are created by organisms in the open-ocean region, and subsequently settle to the seafloor. Hemipelagic sediment contains a pelagic component, generally $5 \%-50 \%$, but up to $75 \%$, with the remainder of the sediment consisting of terrigenous components that enter the ocean at the coast, are carried off the shelf by waves, tidal currents, etc., or in dilute turbidity currents, and eventually settle to the seafloor.) Upper Eocene kaolinitic clay at the base of the pelagic sequence may have been derived from weathered feldspars in exposed volcanic rocks on the ridge.

The unconformities in the sedimentary sequence at Site 828 represent periods of erosion or non-deposition. The absence of uppermost Oligocene and Miocene sediment, as indicated by missing nannofossil Zones CP19 through CN10 between Units II and III, records a time gap of at least $25 \mathrm{~m}$.y. (based on ages of nannofossil zonations shown in Fig. 2A, Staerker [this volume]). This may correlate with an unconformity noted at Site 286 (DSDP) suggesting regional elevation of the seafloor during the Miocene (Collot, Greene, Stokking, et al., 1992). The unconformity between the lower Pliocene ooze (CN11) in Unit II and lower or middle Pleistocene mixed sediment (CN14a) in Unit I represents a time gap of at least 2 m.y.

The abrupt shift in sediment lithology between Units II and I from dominantly pelagic sedimentation to strongly hemipelagic marks the time at which the ridge entered a depositional regime that was influenced by arc-derived turbidity currents. Increasing volcaniclastic input at Site 828 during the Pleistocene is reflected in the upward decrease of carbonate in sediment in the lower part of Unit I and corresponds to increased sedimentation rates. Nannofossil datums marking the first appearance (FAD) of Emiliania huxleyi $(0.28 \pm 0.02 \mathrm{Ma})$ at $49 \mathrm{mbsf}$ and the last appearance (LAD) of Pseudoemiliania lacunosa $(0.46 \pm 0.01 \mathrm{Ma})$ at $57 \mathrm{mbsf}$ suggest that sedimentation rates increased from about $4.4 \mathrm{~cm} / 1000$ years during the middle Pleistocene $(\mathrm{CN} 14 \mathrm{~b})$ to about $18 \mathrm{~cm} / 1000$ years in late Pleistocene time (CN15; Fig. 5). Errors in the ages of nannofossil datums are based on personal communication with Dr. W. Wei, University of California, 1992.

During the roughly $0.50 \mathrm{Ma}$ represented by the Pleistocene section of Site 828 the NDR traveled $65 \mathrm{~km}$ towards the subduction zone based on a convergence rate of about $13 \mathrm{~cm} / \mathrm{yr}$ (Taylor et al., this volume). The increase in proximity to a volcaniclastic sediment source is reflected in the decreasing carbonate content, increasing grain size of the background hemipelagic sediment, and the increase in the frequency of turbidite layers (Fig. 3). The dominant source of sediment for turbidites descending the inner trench slope in this part of the New Hebrides Island Arc is material eroded from Espiritu Santo Island. This large island constitutes part of the Western Belt of the New Hebrides 
Table 1. Total carbonate content of selected samples from Sites 828,827 , and 829 , determined by gasometric analysis.

\begin{tabular}{|c|c|c|c|c|}
\hline Hole/Unit & $\begin{array}{c}\text { Core, section, } \\
\text { interval (cm) } \\
\end{array}$ & $\begin{array}{l}\text { Depth } \\
\text { (mbsf) }\end{array}$ & Lithology & $\begin{array}{c}\mathrm{CaCO} \\
(\%)\end{array}$ \\
\hline \multirow{30}{*}{$\begin{array}{c}\text { Hole 828A } \\
\text { I }\end{array}$} & $1 \mathrm{H}-1,37$ & 0.37 & Silt & 5.78 \\
\hline & $1 \mathrm{H}-3,71$ & 3.71 & Silt & 6.82 \\
\hline & $2 \mathrm{H}-3,120$ & 8.60 & Sandy layer & 8.73 \\
\hline & $2 \mathrm{H}-6,8$ & 11.98 & Sandy layer & 5.79 \\
\hline & $2 \mathrm{H}-6,58$ & 12.48 & Silt & 8.74 \\
\hline & $2 \mathrm{H}-6,108$ & 12.98 & Sandy layer & 6.72 \\
\hline & $3 \mathrm{H}-4,60$ & 19.00 & Sandy layer & 9.31 \\
\hline & $3 \mathrm{H}-4,107$ & 19.47 & Silt & 9.81 \\
\hline & $3 \mathrm{H}-5,94$ & 20.84 & Silt & 9.39 \\
\hline & $4 \mathrm{H}-3,50$ & 26.90 & Ashy layer & 3.46 \\
\hline & $4 \mathrm{H}-3,94$ & 27.34 & Silt & 11.12 \\
\hline & $5 \mathrm{H}-2,29$ & 34.69 & Ashy layer & 7.70 \\
\hline & $5 \mathrm{H}-2,94$ & 35.34 & Silt & 10.63 \\
\hline & $6 \mathrm{H}-6,75$ & 50.65 & Ashy layer & 6.83 \\
\hline & $6 \mathrm{H}-6,94$ & 50.84 & Silt & 16.18 \\
\hline & $7 \mathrm{H}-2,24$ & 53.64 & Silt & 18.50 \\
\hline & $7 \mathrm{H}-2,126$ & 54.66 & Calcareous silt & 25.02 \\
\hline & $7 \mathrm{H}-3,99$ & 55.89 & Calcareous silt & 27.93 \\
\hline & $7 \mathrm{H}-2,126$ & 57.39 & Calcareous silt & 28.64 \\
\hline & $7 \mathrm{H}-5,24$ & 58.14 & Calcareous silt & 32.74 \\
\hline & $7 \mathrm{H}-5,41$ & 58.31 & Mixed sediment & 43.46 \\
\hline & $7 \mathrm{H}-5,61$ & 58.51 & Mixed sediment & 45.09 \\
\hline & $7 \mathrm{H}-5,99$ & 58.89 & Mixed sediment & 52.00 \\
\hline & $7 \mathrm{H}-6,9$ & 59.49 & Mixed sediment & 57.96 \\
\hline & $7 \mathrm{H}-6,64$ & 60.04 & Mixed sediment & 49.78 \\
\hline & $7 \mathrm{H}-6,97$ & 60.37 & Ooze & 78.67 \\
\hline & $7 \mathrm{H}-6,136$ & 60.76 & Mixed sediment & 58.19 \\
\hline & $7 \mathrm{H}-7,18$ & 61.08 & Ooze & 62.65 \\
\hline & $8 \mathrm{H}-1,5$ & 61.45 & Mixed sediment & 30.46 \\
\hline & $8 \mathrm{H}-1,35$ & 61.75 & Mixed sediment & 33.12 \\
\hline \multirow[t]{7}{*}{ II } & $8 \mathrm{H}-1,55$ & 61.95 & Soupy ooze & 90.92 \\
\hline & $8 \mathrm{H}-1,81$ & 62.21 & Soupy ooze & 91.50 \\
\hline & $8 \mathrm{H}-1,99$ & 62.39 & Soupy ooze & 91.56 \\
\hline & $8 \mathrm{H}-1,137$ & 62.77 & Soupy ooze & 90.56 \\
\hline & $8 \mathrm{H}-4,25$ & 66.15 & Soupy ooze & 90.07 \\
\hline & $8 \mathrm{H}-4,99$ & 66.89 & Soupy ooze & 90.19 \\
\hline & $8 \mathrm{H}-6,29$ & 69.19 & Soupy ooze & 86.76 \\
\hline \multirow[t]{12}{*}{ III } & $8 \mathrm{H}-6,62$ & 69.52 & Firm $\infty 0 z e$ & 72.78 \\
\hline & $8 \mathrm{H}-6,123$ & 70.13 & Firm ooze & 77.64 \\
\hline & $9 \mathrm{H}-1,74$ & 71.64 & Firm ooze & 82.65 \\
\hline & $9 \mathrm{H}-3,74$ & 74.64 & Firm ooze & 76.97 \\
\hline & $9 \mathrm{H}-6,74$ & 79.14 & Firm ooze & 81.26 \\
\hline & $10 \mathrm{H}-2,74$ & 82.64 & Firm ooze & 83.83 \\
\hline & $10 \mathrm{H}-2,133$ & 83.23 & Dark layer & 35.24 \\
\hline & $10 \mathrm{H}-2,134$ & 83.24 & Dark layer & 5.99 \\
\hline & $10 \mathrm{H}-2,135$ & 83.25 & Light layer & 41.44 \\
\hline & $10 \mathrm{H}-5,74$ & 87.14 & Firm ooze & 76.13 \\
\hline & $11 \mathrm{H}-1,73$ & 90.63 & Firm ooze & 75.42 \\
\hline & $11 \mathrm{H}-1,88$ & 90.78 & Firm ooze & 69.27 \\
\hline \multirow[t]{3}{*}{ IV } & $11 \mathrm{H}-1,93$ & 90.83 & Matrix cg & 3.72 \\
\hline & $11 \mathrm{H}-2,121$ & 92.61 & Matrix cg & 14.33 \\
\hline & $11 \mathrm{H}-4,33$ & 94.73 & Matrix cg & 3.20 \\
\hline \multirow{14}{*}{$\begin{array}{c}\text { Hole 828B } \\
\text { III }\end{array}$} & $1 \mathrm{R}-1,30$ & 90.30 & Firm ooze & 81.18 \\
\hline & $1 \mathrm{R}-1,81$ & 90.81 & Light layer & 53.34 \\
\hline & $1 \mathrm{R}-1,115$ & 91.15 & Firm ooze & 77.41 \\
\hline & $1 \mathrm{R}-2,115$ & 92.65 & Firm ooze & 74.63 \\
\hline & $1 \mathrm{R}-3,30$ & 93.30 & Firm ooze & 75.41 \\
\hline & $1 \mathrm{R}-3,115$ & 94.15 & Firm ooze & 76.46 \\
\hline & $1 \mathrm{R}-4,115$ & 95.65 & Firm ooze & 73.23 \\
\hline & 1R-5, 23 & 96.23 & Firm ooze & 57.52 \\
\hline & 1R-5, 67 & 96.67 & Clayey $\infty z e$ & 64.67 \\
\hline & $1 \mathrm{R}-5,99$ & 96.99 & Clayey ooze & 56.67 \\
\hline & $1 \mathrm{R}-5,123$ & 97.23 & Clayey ooze & 9.81 \\
\hline & $1 \mathrm{R}-5,143$ & 97.43 & Clayey ooze & 23.56 \\
\hline & $1 \mathrm{R}-6,3$ & 97.53 & Clayey ooze & 17.69 \\
\hline & 1R-6, 7 & 97.57 & Clay & 1.07 \\
\hline
\end{tabular}

\begin{tabular}{|c|c|c|c|c|}
\hline Hole/Unit & $\begin{array}{l}\text { Core, section, } \\
\text { interval (cm) }\end{array}$ & $\begin{array}{l}\text { Depth } \\
\text { (mbsf) }\end{array}$ & Lithology & $\begin{array}{c}\mathrm{CaCO} 3 \\
(\%)\end{array}$ \\
\hline Hole 827A & $2 \mathrm{H}-4,114$ & 15.44 & Silt & 11.05 \\
\hline \multirow[t]{6}{*}{ IA } & $2 \mathrm{H}-4,118$ & 15.48 & Silt & 12.01 \\
\hline & $2 \mathrm{H}-4,121$ & 15.51 & Sandy layer & 10.39 \\
\hline & $3 \mathrm{H}-3,6$ & 22.36 & Silt & 8.55 \\
\hline & $3 \mathrm{H}-3,11$ & 22.41 & Silt & 10.23 \\
\hline & $3 \mathrm{H}-3,15$ & 22.45 & Sandy layer & 11.83 \\
\hline & $3 \mathrm{H}-3,16$ & 22.46 & Sandy layer & 6.61 \\
\hline \multirow[t]{6}{*}{ IB } & $6 \mathrm{H}-4,110$ & 50.50 & Silt & 7.14 \\
\hline & $6 \mathrm{H}-4,111$ & 50.51 & Silt & 9.78 \\
\hline & $6 \mathrm{H}-4,113$ & 50.53 & Sandy layer & 8.42 \\
\hline & $7 \mathrm{H}-3,133$ & 57.13 & Silt & 8.16 \\
\hline & $7 \mathrm{H}-3,137$ & 57.17 & Sandy layer & 5.00 \\
\hline & $7 \mathrm{H}-3,139$ & 57.19 & Sandy layer & 7.84 \\
\hline \multirow[t]{18}{*}{ IC } & $9 \mathrm{H}-3,62$ & 70.62 & Silt & 10.80 \\
\hline & $9 \mathrm{H}-3,80$ & 70.80 & Silt & 10.20 \\
\hline & $9 \mathrm{H}-3,91$ & 70.91 & Sandy layer & 8.26 \\
\hline & $9 \mathrm{H}-3,97$ & 70.97 & Sandy layer & 8.23 \\
\hline & $9 \mathrm{H}-3,105$ & 71.05 & Sandy layer & 1.20 \\
\hline & $9 \mathrm{H}-3,105$ & 71.05 & Sandy layer & 6.42 \\
\hline & $9 \mathrm{H}-3,112$ & 71.12 & Sandy layer & 7.90 \\
\hline & $10 \mathrm{H}-3,54$ & 76.04 & Silt & 9.17 \\
\hline & $10 \mathrm{H}-3,65$ & 76.15 & Silt & 8.96 \\
\hline & $10 \mathrm{H}-3,77$ & 76.27 & Silt & 7.67 \\
\hline & $10 \mathrm{H}-3,91$ & 76.41 & Silt & 6.47 \\
\hline & $10 \mathrm{H}-3,104$ & 76.54 & Silt & 7.85 \\
\hline & $10 \mathrm{H}-3,123$ & 76.73 & Sandy layer & 7.29 \\
\hline & $11 \mathrm{H}-3,141$ & 82.01 & Silt & 7.87 \\
\hline & $12 \mathrm{H}-2,108$ & 85.78 & Silt & 9.47 \\
\hline & $12 \mathrm{H}-3,15$ & 86.35 & Silt & 7.67 \\
\hline & $12 \mathrm{H}-3,64$ & 86.84 & Clay & 10.47 \\
\hline & $13 \mathrm{H}-1,56$ & 88.36 & Sandy layer & 14.82 \\
\hline \multirow[t]{2}{*}{ II } & $13 \mathrm{H}-1,91$ & 88.71 & Silt & 12.46 \\
\hline & $15 X-3,73$ & 104.73 & Silt & 15.59 \\
\hline \multirow[t]{3}{*}{ Hole 827B } & $1 \mathrm{R}-3,75$ & 114.35 & Silt & 11.87 \\
\hline & 2R-3, 69 & 121.29 & Silt & 19.05 \\
\hline & 3R-3, 66 & 130.86 & Silt & 14.87 \\
\hline \multirow[t]{16}{*}{ IIIA } & $4 \mathrm{R}-3,101$ & 140.91 & Light silt & 36.53 \\
\hline & $4 \mathrm{R}-3,146$ & 141.36 & Dark silt & 25.39 \\
\hline & $4 \mathrm{R}-4,5$ & 141.45 & Dark silt & 22.30 \\
\hline & $4 \mathrm{R}-4,11$ & 141.51 & Light silt & 36.06 \\
\hline & $4 \mathrm{R}-6,11$ & 144.51 & Dark silt & 17.89 \\
\hline & $5 \mathrm{R}-2,13$ & 148.13 & Sandy matrix & 35.61 \\
\hline & 5R-2, 42 & 148.42 & Silt & 11.60 \\
\hline & $5 R-2,76$ & 148.76 & Sandy matrix & 32.58 \\
\hline & $5 R-4,28$ & 151.28 & Sandy silt & 30.10 \\
\hline & $6 \mathrm{R}-3,20$ & 159.40 & Silt & 1.78 \\
\hline & 6R-3, 49 & 159.69 & Light ash pod & 2.96 \\
\hline & $6 \mathrm{R}-3,103$ & 160.23 & Dark ashy patch & 4.31 \\
\hline & $6 \mathrm{R}-4,39$ & 161.09 & Light ash layer & 1.60 \\
\hline & $6 \mathrm{R}-4,69$ & 161.39 & Silt & 1.94 \\
\hline & $6 \mathrm{R}-4,123$ & 161.93 & Silt & 18.90 \\
\hline & $9 \mathrm{R}-4,90$ & 190.50 & Silt & 18.09 \\
\hline \multirow[t]{2}{*}{ IIIB } & $11 \mathrm{R}-1,86$ & 205.26 & Chalky clast & 37.33 \\
\hline & $11 \mathrm{R}-1,112$ & 205.52 & Silt clast & 21.21 \\
\hline \multirow[t]{8}{*}{ IIIC } & 13R-1, 80 & 224.50 & Mixed sediment & 41.35 \\
\hline & 13R-3, 40 & 227.10 & Sandy silt & 34.25 \\
\hline & 13R-6, 91 & 232.11 & Light silt & 30.12 \\
\hline & 14R-2, 62 & 235.42 & Chalky layer & 45.15 \\
\hline & 14R-2, 132 & 236.12 & Chalky layer & 56.42 \\
\hline & $14 \mathrm{R}-3,40$ & 236.70 & Dark silt & 3.42 \\
\hline & $15 \mathrm{R}-3,5$ & 246.05 & Sandy silt & 48.56 \\
\hline & 15R-4, 67 & 248.17 & Silt & 16.59 \\
\hline IV & $\begin{array}{l}16 \mathrm{R}-1,10 \\
22 \mathrm{R}-1,85\end{array}$ & $\begin{array}{l}252.70 \\
311.45 \\
\end{array}$ & $\begin{array}{l}\text { Mixed sediment } \\
\text { Matrix cg }\end{array}$ & $\begin{array}{c}46.83 \\
7.51 \\
\end{array}$ \\
\hline
\end{tabular}


Table 1 (continued).

\begin{tabular}{|c|c|c|c|c|}
\hline Hole/Unit & $\begin{array}{l}\text { Core, section, } \\
\text { interval (cm) }\end{array}$ & $\begin{array}{l}\text { Depth } \\
\text { (mbsf) }\end{array}$ & Lithology & $\begin{array}{c}\mathrm{CaCO} 3 \\
(\%)\end{array}$ \\
\hline Hole 829A & $1 \mathrm{R}-2,74$ & 2.24 & Silt & 9.03 \\
\hline \multirow[t]{10}{*}{1} & 2R-2, 74 & 5.04 & Silt & 10.14 \\
\hline & $3 R-2,74$ & 14.54 & Silt & 10.32 \\
\hline & $4 R-2,74$ & 23.84 & Silt & 10.42 \\
\hline & $4 \mathrm{R}-2,107$ & 24.17 & Dark layer & 4.39 \\
\hline & $5 R-2,105$ & 33.55 & Silt & 11.35 \\
\hline & $6 \mathrm{R}-3,74$ & 44.84 & Silt & 19.28 \\
\hline & $7 R-1,74$ & 51.54 & Silt & 19.13 \\
\hline & 7R-1, 110 & 51.90 & Silt & 20.66 \\
\hline & 7R-2, 44 & 52.74 & Silt & 23.61 \\
\hline & 7R-2, 144 & 53.74 & Silt & 36.55 \\
\hline \multirow[t]{4}{*}{ II } & $8 \mathrm{~A}-\mathrm{CC}, 23$ & 60.73 & Chalk & 66.43 \\
\hline & $10 R-1,15$ & 79.85 & Chalk & 86.10 \\
\hline & $12 R-1,5$ & 99.05 & Clay & 15.03 \\
\hline & $12 R-1,32$ & 99.32 & Chalk & 66.06 \\
\hline \multirow[t]{19}{*}{ III } & $12 \mathrm{~A}-2,87$ & 101.37 & Dark layer & 4.99 \\
\hline & $12 R-2,98$ & 101.48 & Silt & 8.62 \\
\hline & $12 R-2,118$ & 101.68 & Cakcareous silt & 22.95 \\
\hline & $13 R-4,98$ & 114.08 & Silt & 14.06 \\
\hline & $13 \mathrm{R}-4,127$ & 114.37 & Chalky lens & 49.66 \\
\hline & $14 \mathrm{R}-5,74$ & 124.94 & Silt & 8.84 \\
\hline & $15 \mathrm{R}-1,97$ & 128.87 & Calcareous silt & 21.15 \\
\hline & 16R-5, 71 & 144.21 & Silt & 16.03 \\
\hline & $17 R-5,115$ & 154.35 & Silt & 15.57 \\
\hline & 18R-6, 12 & 164.42 & Calcareous silt & 21.44 \\
\hline & 18R-6, 34 & 164.64 & Calcareous silt & 24.93 \\
\hline & 18R-6, 41 & 164.71 & Cakcareous silt & 26.59 \\
\hline & 18R-6, 45 & 164.75 & Calcareous silt & 24.53 \\
\hline & 18R-6, 47 & 164.77 & Calcareous silt & 34.98 \\
\hline & 18R-6, 93 & 165.23 & Calcareous silt & 22.37 \\
\hline & 19R-1, 92 & 167.42 & Calcareous silt & 23.83 \\
\hline & $19 R-1,143$ & 167.93 & Calcareous silt & 22.31 \\
\hline & $19 R-1,149$ & 167.99 & Calcareous silt & 25.81 \\
\hline & 19R-2, 105 & 169.05 & Calcareous silt & 30.81 \\
\hline \multirow[t]{2}{*}{ V } & $24 \mathrm{R}-1,70$ & 215.60 & Cg. matrix & 82.93 \\
\hline & $24 \mathrm{R}-1,75$ & 215.65 & Cg. matrix & 77.39 \\
\hline \multirow[t]{4}{*}{ VI } & $43 R-1,14$ & 397.84 & Clayey oze & 55.93 \\
\hline & $43 \mathrm{R}-1,43$ & 398.13 & Clayey odze & 49.81 \\
\hline & $43 R-1,64$ & 398.34 & Clayey ooze & 67.92 \\
\hline & $43 R-1,105$ & 398.75 & Clayey ooze & 62.16 \\
\hline \multirow[t]{3}{*}{ VII } & $43 R-1,124$ & 398.94 & Breccia matrix & 11.38 \\
\hline & $43 R-1,146$ & 399.16 & Breccia matrix & 14.23 \\
\hline & $43 R-3,86$ & 401.56 & Breccia matrix & 1.10 \\
\hline VIII & $44 \mathrm{R}-1,26$ & 407.66 & Chalk & 100.00 \\
\hline \multirow[t]{7}{*}{ IX } & 44R-1, 61 & 408.20 & Mixed sediment & 44.57 \\
\hline & $44 \mathrm{R}-1,80$ & 408.20 & Mixed sediment & 42.50 \\
\hline & $44 \mathrm{R}-1,116$ & 408.56 & Silty chalk & 69.58 \\
\hline & 44R-2, 38 & 409.28 & Silty chalk & 64.72 \\
\hline & $44 \mathrm{R}-2,74$ & 409.64 & Mixed sediment & 54.74 \\
\hline & $45 R-1,18$ & 416.78 & Mixed sediment & 40.52 \\
\hline & $45 R-1,62$ & 417.22 & Calcareous silt & 30.27 \\
\hline
\end{tabular}

Arc platform. Its origin dates back at least to the late Oligocene or early Miocene, when submarine volcanism was associated with a southwest dipping Vitiaz subduction zone (Macfarlane et al., 1988). Since that time, the island has suffered repeated episodes of uplift and subsidence that have intermixed carbonate and volcaniclastic rocks. The most recent phase of uplift is thought to have been initiated by the initial collision of the NDR with the New Hebrides forearc. Late Quaternary uplift has been estimated as several hundred meters based on the dating of raised coral terraces. Using these rates, Taylor (1992) proposed that the total uplift from a typical trench slope depth to 1400 meters above sea level could have taken place in about $2 \mathrm{Ma}$; this time could be reduced to as little as $1 \mathrm{Ma}$ if Taylor's observed Holocene uplift rate of $6 \mathrm{~mm} / \mathrm{yr}$ is applicable to the Pleistocene.

\begin{tabular}{|c|c|c|c|c|}
\hline Hole/Unit & $\begin{array}{l}\text { Core, section, } \\
\text { interval }(\mathrm{cm})\end{array}$ & $\begin{array}{l}\text { Depth } \\
\text { (mbsf) }\end{array}$ & Lithology & $\begin{array}{c}\mathrm{CaCO} \\
(\%)\end{array}$ \\
\hline IX & $\begin{array}{l}45-1,94 \\
45 R-1,115\end{array}$ & $\begin{array}{l}417.54 \\
417.75\end{array}$ & $\begin{array}{l}\text { Mixed sediment } \\
\text { Chalk }\end{array}$ & $\begin{array}{l}39.21 \\
89.95\end{array}$ \\
\hline$x$ & $\begin{array}{l}46 \mathrm{~A}-1,20 \\
47 \mathrm{~A}-1,27 \\
47 \mathrm{R}-1,50 \\
47 \mathrm{R}-1,58\end{array}$ & $\begin{array}{l}421.80 \\
426.87 \\
427.10 \\
427.18\end{array}$ & $\begin{array}{l}\text { Chalk } \\
\text { Chalk } \\
\text { Brown clay } \\
\text { Chalky clay }\end{array}$ & $\begin{array}{l}92.04 \\
84.53 \\
12.13 \\
25.53\end{array}$ \\
\hline$\overline{X I}$ & $\begin{array}{l}47 \mathrm{R}-1,74 \\
47 \mathrm{R}-1,100 \\
48 \mathrm{R}-1,14 \\
48 \mathrm{R}-1,45 \\
48 \mathrm{R}-1,95\end{array}$ & $\begin{array}{l}427.34 \\
427.60 \\
436.44 \\
436.75 \\
437.25 \\
\end{array}$ & $\begin{array}{l}\text { Mixed sediment } \\
\text { Mixed sediment } \\
\text { Mixed sediment } \\
\text { Chalk } \\
\text { Mixed sediment }\end{array}$ & $\begin{array}{l}4.93 \\
45.09 \\
53.70 \\
83.72 \\
36.64\end{array}$ \\
\hline XII & $\begin{array}{l}49 \mathrm{R}-\mathrm{CC}, 9 \\
49 \mathrm{R}-\mathrm{CC}, 21 \\
50 \mathrm{R}-1,33 \\
51 \mathrm{R}-1,16 \\
51 \mathrm{R}-1,80 \\
51 \mathrm{R}-2,5 \\
51 \mathrm{R}-2,58 \\
51 \mathrm{R}-2,97 \\
51 \mathrm{R}-2,135 \\
51 \mathrm{R}-3,3\end{array}$ & $\begin{array}{l}445.99 \\
446.11 \\
445.93 \\
460.26 \\
460.90 \\
461.65 \\
462.18 \\
462.57 \\
462.95 \\
463.13\end{array}$ & $\begin{array}{l}\text { Chalk } \\
\text { Chalk } \\
\text { Chalk } \\
\text { Clayey chalk } \\
\text { Clayey chalk } \\
\text { Clayey chalk } \\
\text { Clayey chalk } \\
\text { Clayey chalk } \\
\text { Clayey chalk } \\
\text { Clayey chalk }\end{array}$ & $\begin{array}{l}95.32 \\
77.67 \\
90.10 \\
45.22 \\
23.35 \\
29.05 \\
60.72 \\
58.47 \\
44.75 \\
51.89\end{array}$ \\
\hline XIII & $\begin{array}{l}52 \mathrm{R}-1,29 \\
52 \mathrm{R}-1,39 \\
52 \mathrm{R}-1,87 \\
52 \mathrm{R}-1,137 \\
52 \mathrm{R}-\mathrm{CC}, 7 \\
53 \mathrm{R}-1,50 \\
53 \mathrm{R}-1,148\end{array}$ & $\begin{array}{l}465.49 \\
465.59 \\
466.07 \\
466.57 \\
467.20 \\
475.30 \\
476.28\end{array}$ & $\begin{array}{l}\text { Sandstone } \\
\text { Sandstone } \\
\text { Chalk } \\
\text { Silty sandstone } \\
\text { Silty sandstone } \\
\text { Silty sandstone } \\
\text { Silty sandstone }\end{array}$ & $\begin{array}{c}22.74 \\
59.48 \\
79.90 \\
9.09 \\
12.99 \\
24.78 \\
20.13\end{array}$ \\
\hline XIV & $\begin{array}{l}54 \mathrm{~A}-1,5 \\
54 \mathrm{R}-1,30 \\
55 \mathrm{R}-1,42 \\
55 \mathrm{R}-1,88 \\
55 \mathrm{R}-1,104 \\
55 \mathrm{R}-1,117 \\
55 \mathrm{R}-1,125 \\
55 \mathrm{R}-\mathrm{CC}, 10\end{array}$ & $\begin{array}{l}484.55 \\
484.80 \\
494.52 \\
494.98 \\
495.14 \\
495.26 \\
495.35 \\
495.51\end{array}$ & $\begin{array}{l}\text { Chalk } \\
\text { Chalk } \\
\text { Mixed sediment } \\
\text { Mixed sediment } \\
\text { Mixed sediment } \\
\text { Ooze layer } \\
\text { Mixed sediment } \\
\text { Chalk }\end{array}$ & $\begin{array}{l}98.38 \\
86.03 \\
53.73 \\
64.41 \\
32.74 \\
84.55 \\
60.23 \\
82.17\end{array}$ \\
\hline$\overline{X V}$ & $\begin{array}{l}56 \mathrm{R}-1,141 \\
56 \mathrm{R}-2,5 \\
56 \mathrm{R}-2,129 \\
56 \mathrm{R}-3,79 \\
57 \mathrm{R}-1,50 \\
57 \mathrm{R}-2,50 \\
57 \mathrm{R}-3,50 \\
57 \mathrm{R}-3,70\end{array}$ & $\begin{array}{l}505.21 \\
505.35 \\
506.59 \\
507.59 \\
514.00 \\
515.50 \\
517.00 \\
517.20\end{array}$ & $\begin{array}{l}\text { Chalk layer } \\
\text { Chalk layer } \\
\text { Chalk layer } \\
\text { Sandy siltstone } \\
\text { Sandy siltstone } \\
\text { Sandy siltstone } \\
\text { Sandy siltstone } \\
\text { Sandy siltstone }\end{array}$ & $\begin{array}{l}73.97 \\
90.31 \\
65.22 \\
11.67 \\
16.60 \\
11.37 \\
25.12 \\
66.71\end{array}$ \\
\hline XVI & $\begin{array}{l}57 R-3,74 \\
57 R-4,11 \\
57 R-4,15 \\
58 R-1,21 \\
60 R-1,40 \\
63 R-1,61 \\
64 R-1,5\end{array}$ & $\begin{array}{l}517.24 \\
518.11 \\
518.15 \\
523.41 \\
543.00 \\
572.11 \\
581.25\end{array}$ & $\begin{array}{l}\text { Brown clay } \\
\text { Cg. matrix } \\
\text { Cg. matrix } \\
\text { Cg. matrix } \\
\text { Cg. matrix } \\
\text { Cg. matrix } \\
\text { Cg. matrix }\end{array}$ & $\begin{array}{c}13.10 \\
65.37 \\
48.79 \\
1.62 \\
19.50 \\
1.75 \\
2.79\end{array}$ \\
\hline
\end{tabular}

The dramatic and recent uplift of Espiritu Santo Island would have had a profound effect on the sediment being supplied to the forearc area. Emergence of the island above sea level exposed late Oligocene to Pleistocene carbonate and volcaniclastic rocks to subaerial erosion processes, thus creating a robust source of epiclastic sediment for the forearc region. This sediment would have been preferentially distributed to the west of Espiritu Santo Island by the prevailing westwardflowing South Equatorial Current and Trade Drift Convergence. Storm and seismically triggered turbidity currents would provide a mechanism for transferring this material to the deep water environment.

In addition to tectonic control, sediment supply was also likely to be modulated by Pleistocene sea level changes, which caused relatively rapid transgressive-regressive cycles. Low stands of sea level would 
Hole

828A Layers/section (N)
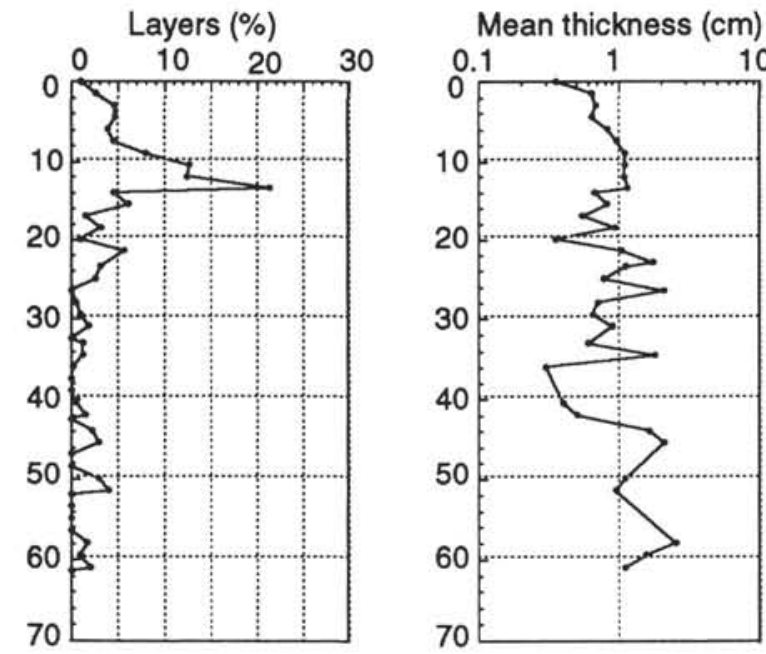

Max. thickness $(\mathrm{cm})$
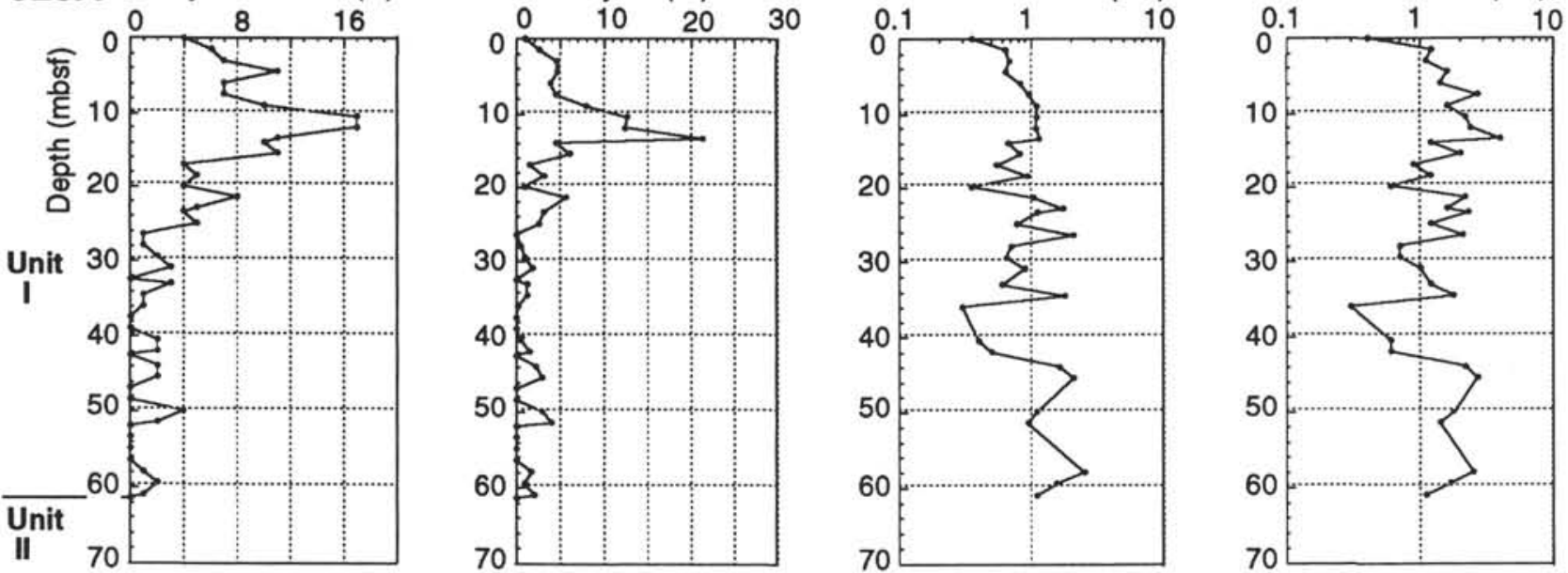

\section{Hole}

827 A Layers/section (N)

Layers (\%)

Mean thickness $(\mathrm{cm})$

Max. thickness $(\mathrm{cm})$
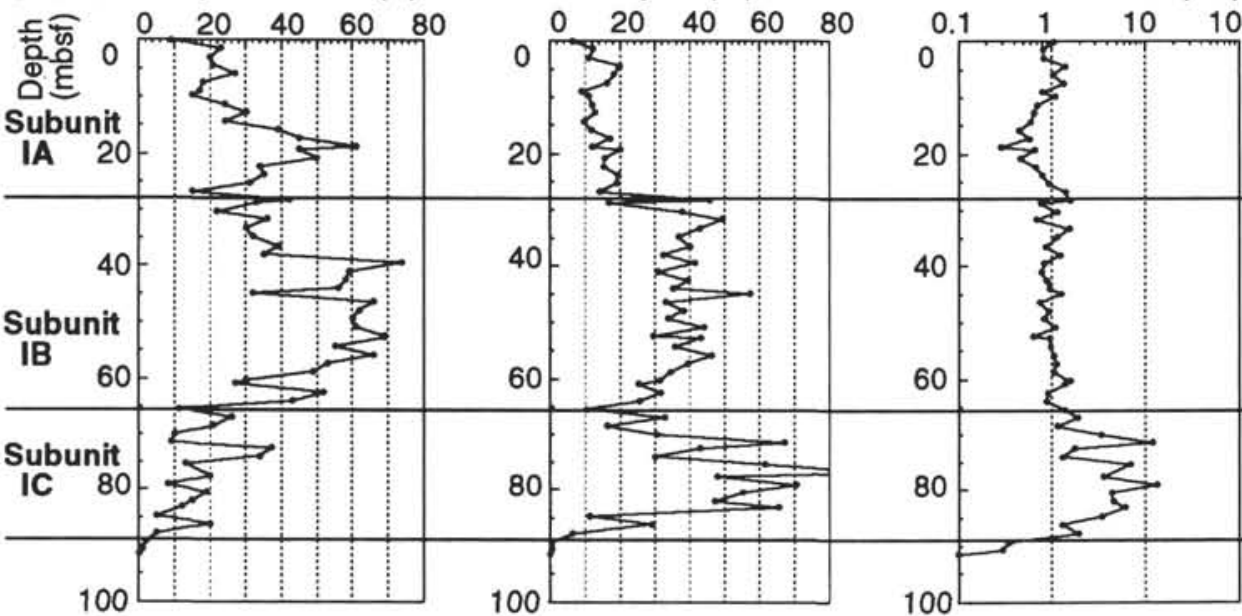

.

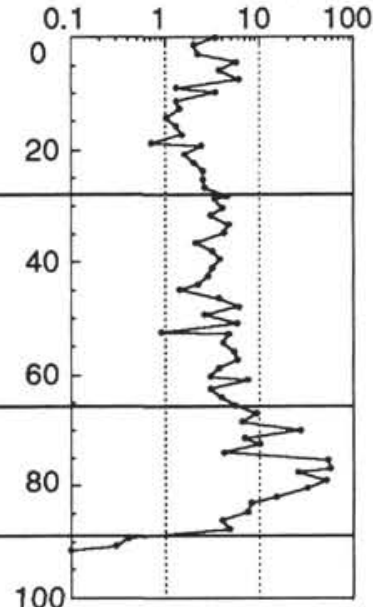

Hole

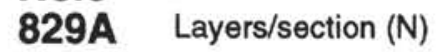

100
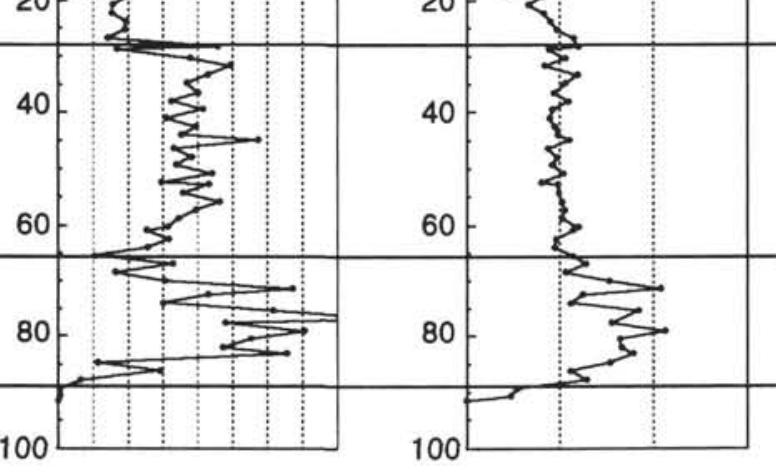

100
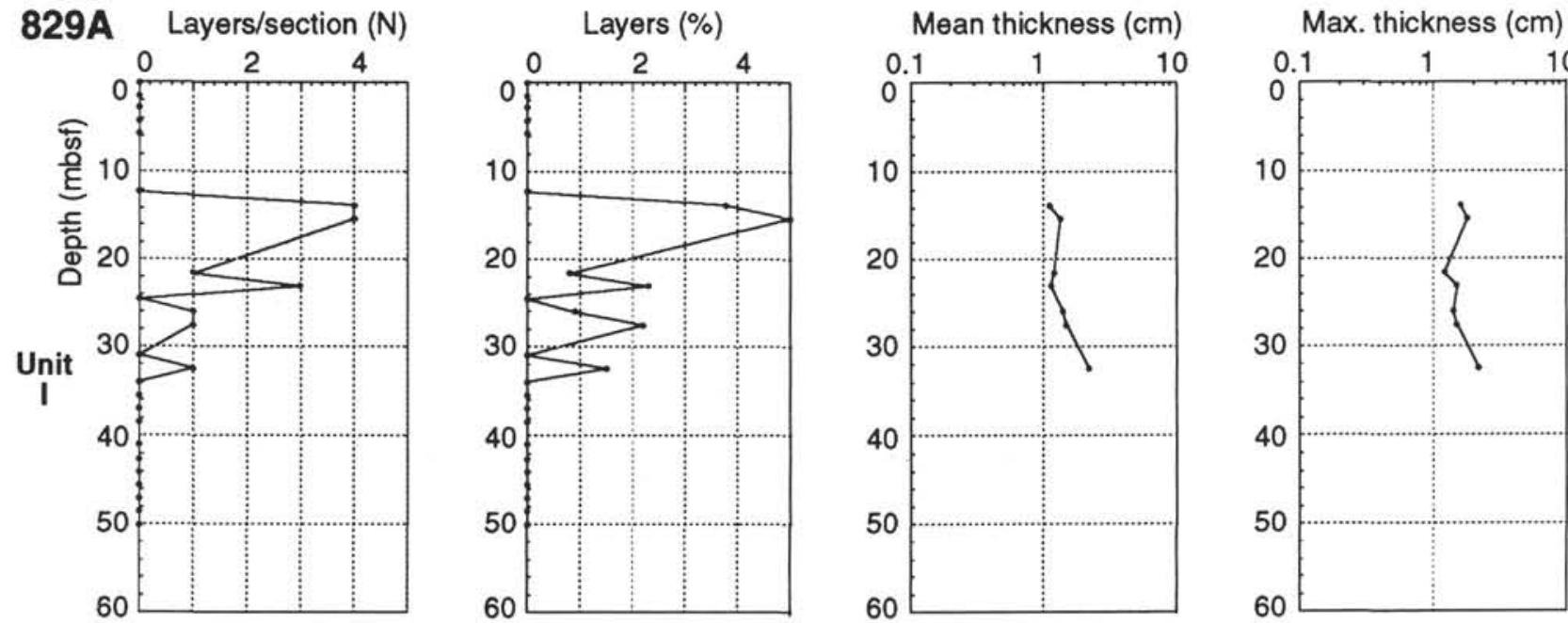

Figure 3. Plots showing the occurrence of crystal/ithic and glass-rich layers in the upper parts of Holes $827 \mathrm{~A}, 828 \mathrm{~A}$, and $829 \mathrm{~A}$. The variables plotted are, from left to right, the number of layers per section, the percentage of each section represented by layers, the mean thickness of the layers in each section, and the maximum thickness of the layers in each section. Note change of scales between sites. 

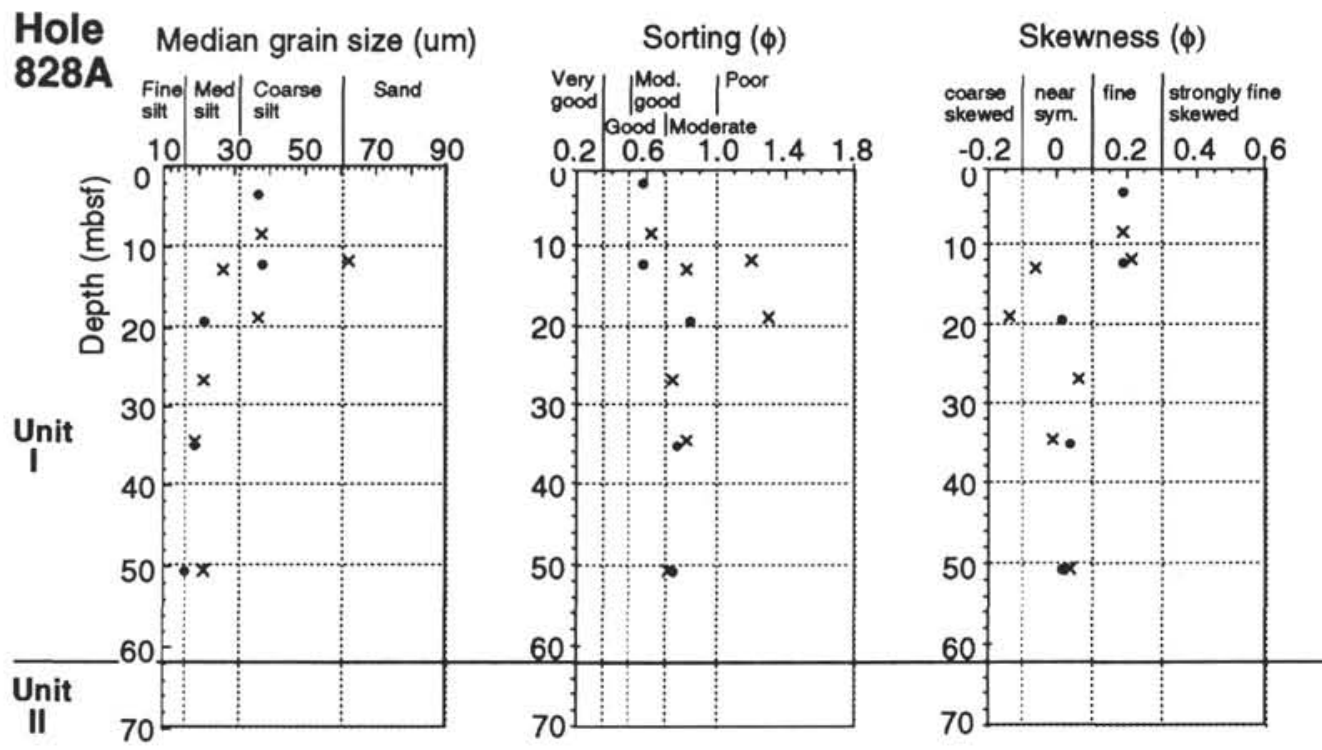

$\mathrm{CaCO}_{3}(\%)$
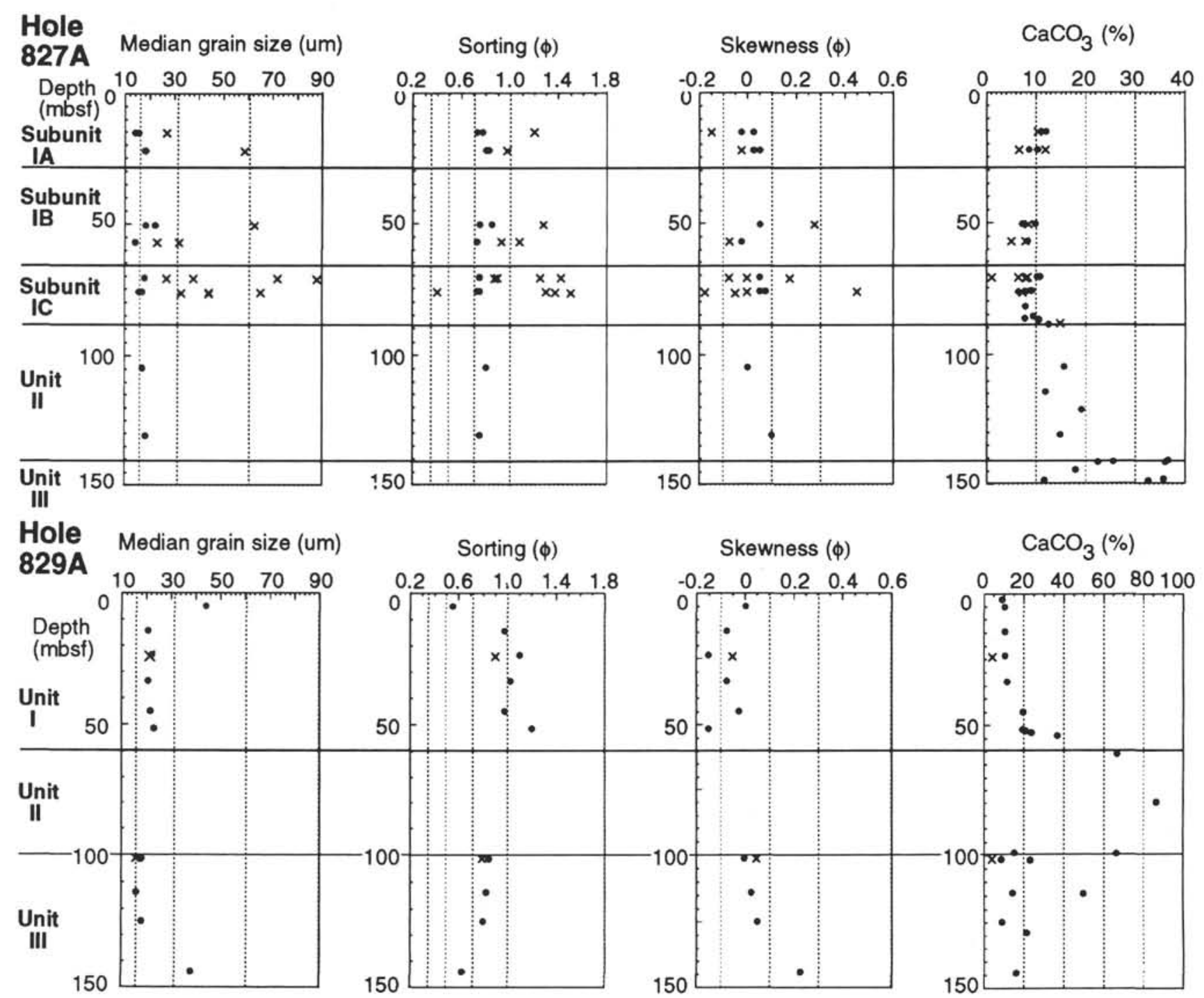

Figure 4. Plots showing median grain size, sorting, skewness, and total carbonate content in the upper parts of Holes 827A, 828A, and 829A. Samples from crystal/lithic and glass-rich layers are marked with an "X." 
have moved local sediment inputs seaward of the island and exposed sediments on the narrow shelves to processes of subaerial erosion. These conditions would favor the enhanced generation of turbidity currents to the forearc environment. Shanmugam and Moiola (1982) have previously demonstrated a strong correlation between low sea level stands and increased frequency of turbidite deposits.

The signature of Pleistocene glacial-interglacial cycles at Site 828 has been examined by Martinez (this volume) using the proxy indicator of dissolution cycles in foraminifera. He recognized five dissolution cycles within the $0-60 \mathrm{mbsf}$ sequence of $828 \mathrm{~A}$. Like dissolution cycles of the Ontong Java Plateau, these cycles are out of phase with the oxygen isotope $\left(\delta^{18} \mathrm{O}\right)$ record. Accelerator mass spectrometer (AMS) ${ }^{14} \mathrm{C}$ dating constrains the offset from the last glacial maximum to be about 8 k.y. Based on foraminiferal distribution, Martinez (this volume) assigned ages of 18 k.y., 120 k.y., and 250 k.y. to depths of 12 , 45 , and $58 \mathrm{mbsf}$, respectively, in Unit I of Hole 828A. These inferred ages are younger than the ages suggested by the nannofossil zonation of Unit I and result in higher inferred sedimentation rates (Fig. 5). Reasons for the differences between foraminiferal and nannofossil age zonation of Unit I are uncertain, but Martinez (written communication to Staerker, 1991) offers two explanations. First, he points out that a major dissolution peak (determined by fragmentation and low percent of solution susceptible species) occurs below the proposed FAD of $E$. huxleyi at 49 mbsf. As $E$. huxleyi is susceptible to dissolution and occurs in low numbers at the beginning of its evolutionary appearance, Martinez suggests that the apparent FAD of $E$. huxleyi may be too high. Second, he suggests that the LAD of $P$. lacunosa may be artificially high because of reworking. The differences between foraminiferal and nannofossil zonations could be resolved with oxygen isotope stratigraphy, but such analyses are not presently available for Site 828 .

The sedimentation rates derived from the foraminiferal zonation of Hole 828A and AMS dating (Martinez, this volume) suggest that the peak in turbidite frequency that occurs at 12 mbsf in Unit I (Fig. 3) coincides with the last glacial maximum, at 18,000 yrs. The lack of additional peaks suggesting increased turbidite frequency during earlier glacial maxima at Site 828 probably reflects the more distal location of the site relative to the subduction front in earlier Pleistocene time.

Despite the fact that Site 828 moved progressively towards the source area of volcaniclastic sediment (New Hebrides Island Arc) during the Pleistocene, the site remained elevated relative to the trace of the subduction zone. Site 828 is currently $150 \mathrm{~m}$ above the bathymetric low that separates the NDR from the inner trench slope. Deposition of turbidites at Site 828 thus indicates either upslope flow of turbidity currents or currents with thicknesses of $>150 \mathrm{~m}$. Upslope flow of turbidites has been documented in a number of convergent margin settings (Muck and Underwood, 1990; Dolan et al., 1989). If deposition was caused primarily by run-up of currents descending from the inner trench slope, their thickness would need to be about $100 \mathrm{~m}$ (Muck and Underwood, 1990). It is, of course, possible that deposition was the result of thick flows (>150 m). For example, Dolan et al. (1990) have suggested that some turbidity currents attain a thickness of $>1500 \mathrm{~m}$.

Episodic volcanic eruptions in the vicinity of Site 828 during middle and late Pleistocene time are suggested by the volcanic glassrich layers in the lower $40 \mathrm{~m}$ of Unit I. Geochemical analyses of the ash suggests a source in the Central Chain, $150 \mathrm{~km}$ to the southeast (Coltorti et al., this volume; Baker et al., this volume).

\section{Site 827}

Site 827 is located on an elevated terrace of the forearc slope that is bounded to the north by a steep scarp. This elevated forearc area is aligned with the trace of the NDR as it impinges on the forearc slope from the west. Upslope from Site 827 is a broad shallow area known as Wousi Bank. The site is $4 \mathrm{~km}$ east of the trace of the subduction
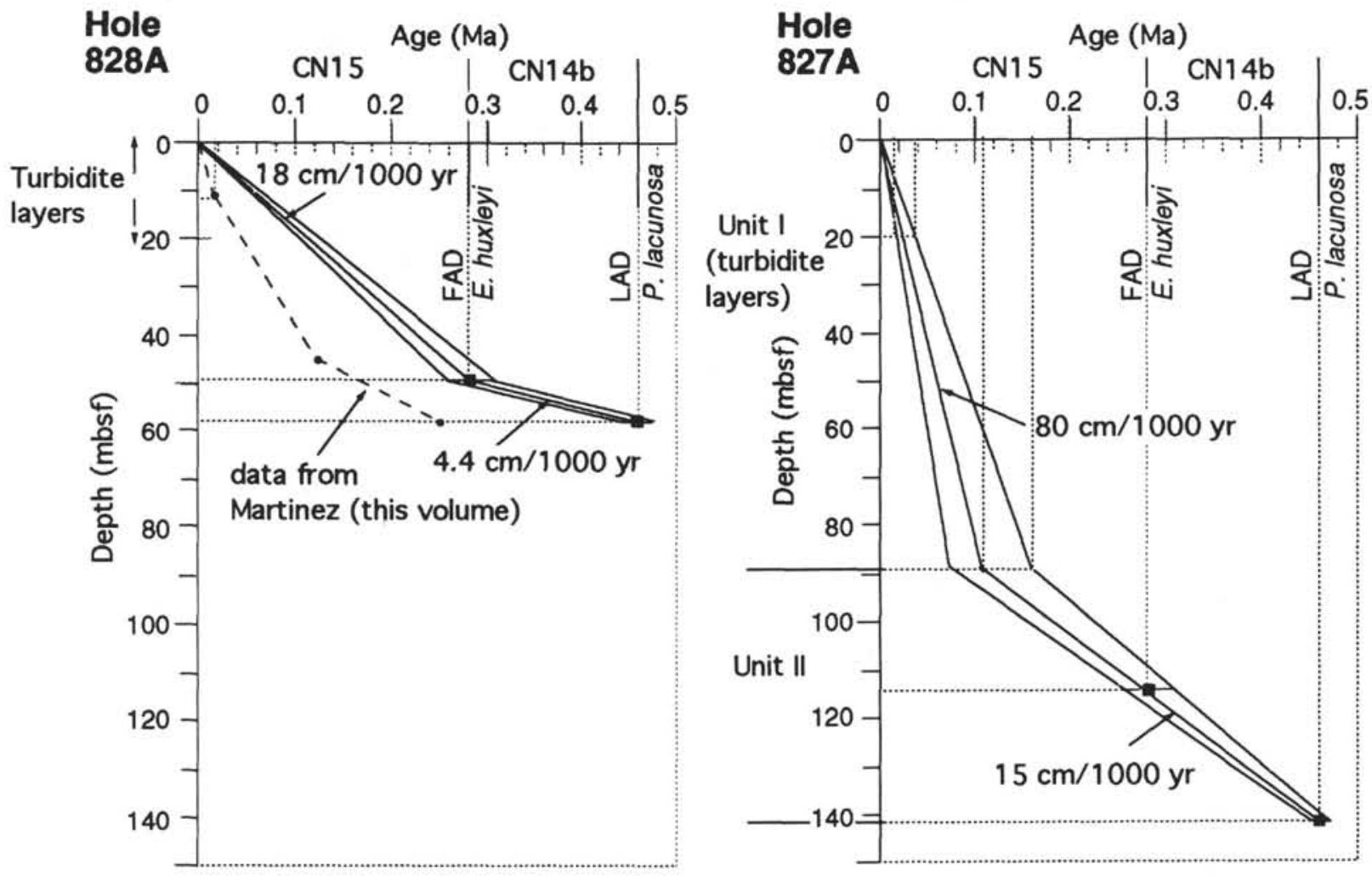

Figure 5. Sedimentation rates for the past 0.5 m.y. at Sites 828 and 827 based on the first appearance of $E$. huxleyi $0.28 \pm 0.02 \mathrm{Ma}$ and the last appearance of $P$. lacunosa $0.46 \pm 0.01 \mathrm{Ma}$. For Site 828 we have also plotted the foraminiferal data of Martinez (this volume), indicated by the solid circles and dashed line. For Site 827 sedimentation is assumed to have remained constant throughout the deposition of Unit II, because carbonate contents are relatively uniform in this interval, ranging from $10 \%$ to $20 \%$ (see Fig. 4). 


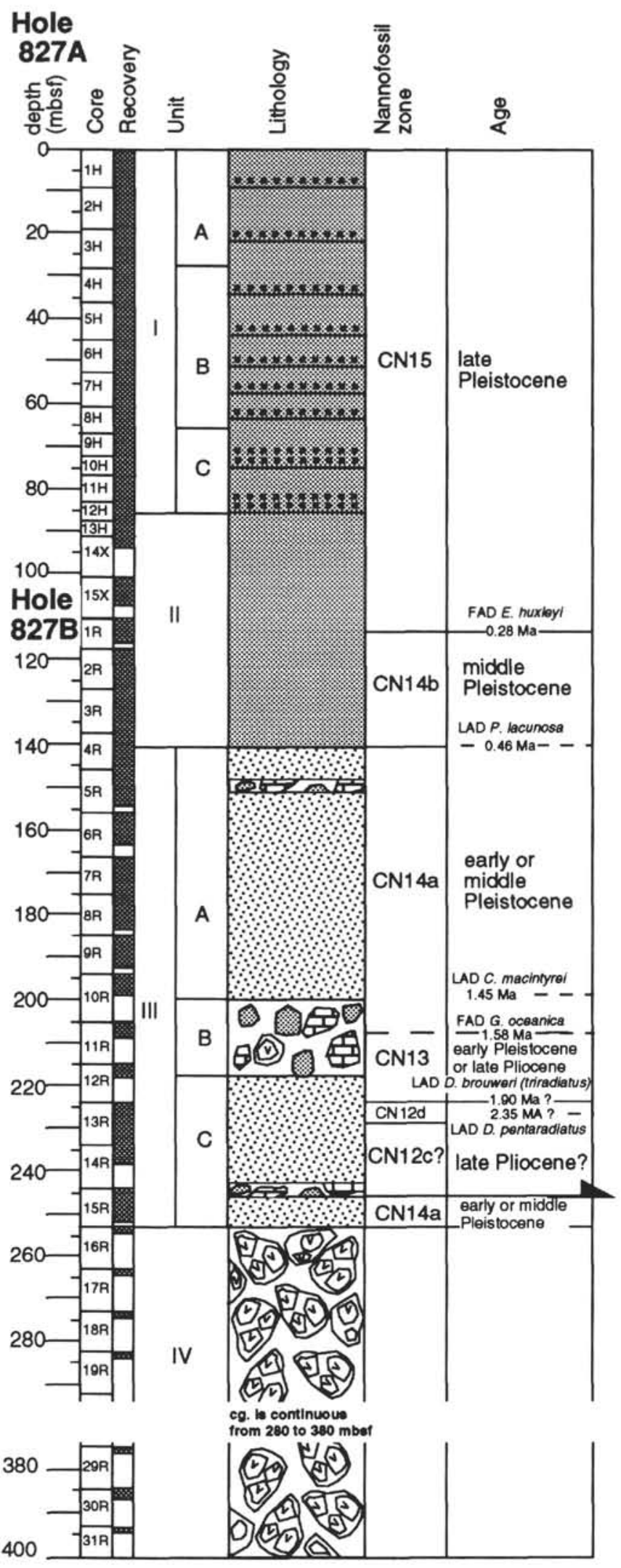

Figure 6. Revised lithostratigraphy for Site 827. zone and $35 \mathrm{~km}$ west of the coast of Espiritu Santo Island. Unlike Site 828 , Site 827 has not suffered significant lateral translation during the time represented by its sedimentary sequence. Drilling at Site 827 penetrated $252 \mathrm{~m}$ of upper Pliocene to upper Pleistocene marine sediment overlying $148 \mathrm{~m}$ of sed-lithic conglomerate of unknown age; this sediment was subdivided into four lithostratigraphic units (Units I-IV). Additions and modifications to shipboard descriptions of the units are presented below; a revised lithostratigraphic column for Site 827 is shown in Figure 6.

Unit I (0-88.4 mbsf; Sections $134-827 \mathrm{~A}-1 \mathrm{H}, 0 \mathrm{~cm}$, to $-13 \mathrm{H}-1,60$ $\mathrm{cm}$ ) is composed of upper Pleistocene (CN15) volcanic silt with layers of normally graded silty sand. These layers are composed dominantly of volcanic crystals and lithic fragments. They are interpreted as epiclastic deposits emplaced by storm or seismically generated turbidity currents. The base of Unit I is placed at $88.4 \mathrm{mbsf}$, rather than at 86 mbsf as defined aboard ship, to include sandy layers that occur between 86 and 88.4 mbsf.

Unit I is divided into three subunits on the basis of the frequency and thickness of the turbidite layers (Fig. 3). In Subunit IA (0-27 mbsf; Sections $134-827 \mathrm{~A}-1 \mathrm{H}, 0 \mathrm{~cm}$, to $-3 \mathrm{H}-6,150 \mathrm{~cm})$, the number of layers per section ranges from 9 to 61 (average $=30$ ), the mean thickness of the layers ranges from 0.3 to $1.5 \mathrm{~cm}$ (average $=1 \mathrm{~cm}$ ), and the maximum thickness of an individual layer is $6 \mathrm{~cm}$. In Subunit IB (27-66 mbsf; Sections $134-827 \mathrm{~A}-3 \mathrm{H}-6,150 \mathrm{~cm}$, to $-8 \mathrm{H}-4$, at 50 $\mathrm{cm}$ ) the number of layers per section ranges from 22 to 74 (average $=47$ ), the mean thickness of the layers ranges from 0.6 to $1.6 \mathrm{~cm}$ (average $=1 \mathrm{~cm}$ ), and the maximum thickness of an individual layer is $7.5 \mathrm{~cm}$. In Subunit IC (66-88.4 mbsf; Sections 134-827A-8H-4, 50 $\mathrm{cm}$, to $-13 \mathrm{H}-1,60 \mathrm{~cm}$ ), the number of layers per section ranges from 3 to 37 (average $=16$ ), mean thickness of the layers ranges from 1.1 to 13.2 (average $=4.2 \mathrm{~cm}$ ), and the maximum thickness of an individual layer is $57 \mathrm{~cm}$. Although the number of layers per section is greatest in Subunit IB, the layers are thickest in Subunit IC, and the proportion of each section represented by turbidite layers decreases upward throughout Unit I (see Fig. 3).

The turbidite layers and intervening silt intervals in Subunits IA, IB, and IC are similar in composition, consisting predominantly of volcanic minerals such as plagioclase, pyroxenes, and opaque minerals including magnetite; volcanic glass is rare in these deposits. Carbonate content ranges from $6 \%$ to $12 \%$ in the silt and from $1 \%$ to $15 \%$ in the sandy layers (Table 1 and Fig. 4). The mean grain size of the silt ranges from 14 to $22 \mu \mathrm{m}$, sorting is moderate, and the sediment has a near symmetrical size distribution (Fig. 4). X-radiographs show no evidence of layering within the silt. The sandy layers fine upward and are generally poorly sorted with variable skewness; the bases of the sandy layers are sharply defined.

Unit II (88.4-141 mbsf; Sections 134-827A-13H-1, $60 \mathrm{~cm}$, to $-4 \mathrm{R}-3,90 \mathrm{~cm}$ ) is characterized by an absence of sandy turbidite layers and consists entirely of upper and middle Pleistocene (CN15 and $\mathrm{CN} 14 \mathrm{~b}$ ) volcanic silt. The mean grain size of the silt is 17 to $18 \mu \mathrm{m}$, sorting is moderate, and the sediment has a near symmetrical grain size distribution. Carbonate content is generally higher than in Unit I, ranging from $10 \%$ to $20 \%$.

Sediment in Unit III (141-252 mbsf; Sections 134-827B-4R-3, 90 $\mathrm{cm}$, to $-15 \mathrm{R}-\mathrm{CC}, 15 \mathrm{~cm}$ ) is typically coarser than that in Unit II, consisting mainly of lower or middle Pleistocene (CN14a) to upper Pliocene $(\mathrm{CN} 12 \mathrm{c})$ partially-lithified sandstone, sandy siltstone, and conglomerate. Clasts in the conglomerate that were described aboard ship as biogenic and pelagic limestone are redesignated as chalk and recognized as important components of the unit. In contrast to sediment in Units I and II, which is relatively undeformed, sediment in Units III is highly sheared and fractured (see Meschede and Pelletier, this volume). A biostratigraphic age inversion near the base of Unit III, defined by a 
reoccurrence of the nannofossil Gephyrocapsa oceanica, which first occurs at $1.58 \mathrm{Ma}$, places upper Pliocene sediment in nannofossil Zone $\mathrm{CN} 12 \mathrm{c}$ over early Pleistocene sediment in Zone CN14a.

Unit III is divided into 3 subunits. Subunit IIIA (141-200 mbsf; Sections 134-827B-4R-3, $90 \mathrm{~cm}$, to $-10 \mathrm{R}-4,120 \mathrm{~cm}$ ) consists of lower or middle Pleistocene (CN14a), partially lithified sandy to clayey volcanic siltstone with intervals of breccia and conglomerate. The upper $7 \mathrm{~m}$ of the section is brecciated, with angular clasts of sandy siltstone, $1 \mathrm{~mm}$ to $3 \mathrm{~cm}$ in size, in a matrix of similar lithology. From 148 to 150 mbsf (Sections 134-827B-5R-2, $35 \mathrm{~cm}$, to $-5 \mathrm{R}-3,82 \mathrm{~cm}$ ), the section consists primarily of matrix-supported conglomerate with clasts of siltstone, chalk, and rare highly lithified sedimentary rock fragments. The matrix of the breccia is early or middle Pleistocene $(\mathrm{CN} 13 \mathrm{~b} / \mathrm{CN} 14 \mathrm{a})$ in age, one of the silt clasts is late Pliocene (CN12d) and one of the chalk clasts is middle Eocene (CP12a). From 150 to $170 \mathrm{mbsf}$ (Sections $134-827 \mathrm{~B}-5 \mathrm{R}-3,82 \mathrm{~cm}$, to $-7 \mathrm{R}-3,150 \mathrm{~cm}$ ), isolated pumice fragments and pods of light-gray ash occur in a section of interbedded mud, silt, and sand. A 5-cm-thick layer of volcanic conglomerate occurs at 167.7 mbsf (Section 134-827B-7R-2, $42 \mathrm{~cm}$ ).

Subunit IIIB (200-218 mbsf; Section 134-827B-10R-4, $120 \mathrm{~cm}$, to $-12 \mathrm{R}-3,90 \mathrm{~cm}$ ) consists of lower Pleistocene and possibly upper Pliocene $(\mathrm{CN} 13)$ chalk-siltstone breccia with angular clasts of chalk and siltstone, 0.1 to $5 \mathrm{~cm}$ in size, and, less commonly, coral fragments and highly lithified pieces of sandstone and andesite breccia, which are tightly packed in a sandy matrix. The siltstone clasts are late Pliocene and early Pleistocene (CN12d, CN12d/13a, CN13a, CN13b/14a) in age; the chalk clasts are early and middle Eocene (CP11 and CP12) in age.

Subunit IIIC (218-252 mbsf; Sections 134-827B-12R-3, 90 cm, to $-15 \mathrm{R}-\mathrm{cc}, 15 \mathrm{~cm}$ ) consists of partially lithified upper Pliocene and possibly lower Pleistocene (CN12c, CN12d, and CN13) foraminiferal siltstone with muddy intervals and thin layers of ash and mixed sediment. Rare, highly lithified, dark rock fragments $1-2 \mathrm{~mm}$ in size occur throughout the upper $10 \mathrm{~m}$ of the section. A conglomerate occurs in the interval from 244.3 to 247.2 mbsf, with clasts of chalk (that have not been dated), sandstone, and highly lithified rock fragments. The biostratigraphic inversion that places nannofossil Zone $\mathrm{CN} 12 \mathrm{c}$ over Zone CN14a occurs at the base of the conglomerate.

Unit IV (252-400.4 mbsf; Sections 134-827B-15R-CC, $15 \mathrm{~cm}$, to $-31 \mathrm{R}-\mathrm{CC}$ ) consists of partially lithified, matrix-supported, sed-lithic conglomerate; the interval is barren of microfossils and, like Unit III, Unit IV is highly tectonized. The clasts in the conglomerate are highly lithified pieces of andesite breccia, volcanic siltstone, and sandstone containing abundant plagioclase, pyroxene, and amphibole crystals.

\section{Sedimentologic History}

Collot, Greene, Stokking, et al. (1992) suggested that the relatively undeformed sediment in the upper $152 \mathrm{~m}$ of Site 827 (Units I and II) was deposited on the forearc slope, but these authors were ambivalent about the origin of the highly tectonized sediment below $152 \mathrm{mbsf}$ (Units III and IV). They suggested that the tectonized sediment "could have been deposited on the arc slope and subsequently deformed, or could have been deposited in the central New Hebrides Trench and then accreted to the arc slope as imbricated thrust sheets" (Collot, Greene, Stokking, et al., 1992, p. 127). Our data, which identifies a biostratigraphic inversion indicative of a thrust fault near the base of Unit III, provides evidence that Unit IV and the base of Unit III are accreted.

The presence of andesitic clasts and amphiboles in the sed-lithic conglomerate comprising Unit IV suggests that these rocks were derived from the arc, rather than from the ridge. Therefore Unit IV is interpreted as a debris flow of arc material that slid into the trench or onto a flat spot of the ridge and was later accreted onto the forearc slope. Accretion of Unit IV and sediment at the base of Unit III is thought to have occurred within the past $1.58 \mathrm{~m}$.y. because the accreted sediment at the base of Unit III contains G. oceanica, which first appeared 1.58 m.y. ago.

The origin of the highly tectonized sediment in Unit III above the biostratigraphic inversion remains uncertain, but based on the lack of paleontologic evidence for a thrust fault, it is interpreted as an in-situ slope deposit. The abundance of middle and lower Eocene chalk clasts in the conglomerates of Unit III suggest the erosion of exotic chalk accreted to the arc slope prior to the deposition of Unit III, as the oldest rocks known in the Western Belt Islands are Oligocene mudstones (Macfarlane et al., 1988). Although lower and middle Eocene sediment was not recovered at Site 828 on the NDR or at Site 831 on the Bougainville Guyot (Collot, Greene, Stokking, et al., 1992), middle Eocene carbonate has been dredged from the southern part of the d'Entrecasteaux Zone (DEZ) on Bougainville Guyot (Collot et al., 1992).

In-situ sedimentation of sandy siltstone and conglomerate on the forearc slope at Site 827 changed to deposition of hemipelagic silt (Unit II) about 460,000 years ago. Nannofossil datums FAD $E$. huxleyi $(0.28 \pm 0.02 \mathrm{Ma})$ at $114 \mathrm{mbsf}$ and LAD P. lacunosa $(0.46 \pm$ $0.01 \mathrm{Ma})$ at $141 \mathrm{mbsf}$ suggest that the rate of deposition of hemipelagic silt during middle Pleistocene Zone $\mathrm{CN} 14 \mathrm{~b}$ was about 15 $\mathrm{cm} / 1000$ yr (Fig. 5).

The fining upward grain sizes, sharply defined bases, and relative thinness of the layers in Unit I at Site 827 suggest that, like the sandy layers at Site 828, the layers at Site 827 are distal turbidite deposits. The silt intervals between the sandy layers at Site 827 are interpreted as hemipelagic sediment, rather than the upper parts of the turbidite layers as suggested by Collot, Greene, Stokking, et al. (1992), because the silts are relatively uniform in grain size, showing no indication of fining upward, and they are similar in size, sorting, and skewness to hemipelagic sediment in Unit II. Moreover, no evidence is present of internal layering in the silt, and no apparent correlation exists between the thickness of sandy layers and overlying silt intervals.

The nature of turbidite deposition changes dramatically within Unit I as shown by the plots of layer frequency, thickness, and percentage of each section represented by layers (Fig. 3). Subunit IA shows variable frequency of turbidites and an inverse correlation of frequency with bed thickness. Thus, despite a peak in turbidite frequency at about $20 \mathrm{mbsf}$, net turbidite accumulation does not increase because the individual beds are very thin. This is shown in the plot of percent layers (turbidites) as a function of depth. In Subunit IA, the proportion of each section represented by layers remains relatively constant, between $10 \%$ and $20 \%$ (Fig. 3). Subunit IB is marked by a broad peak in turbidite frequency and a sharp increase in the accumulation rate of turbidites (layers form approximately $40 \%$ of sections in this subunit). Subunit IC differs from Subunits IA and IB in that the frequency of turbidites is generally low, but the mean thickness of the layers is up to an order of magnitude larger than in the other two subunits. This is reflected in the high proportion of layers (layers form approximately $60 \%$ of each section; Fig. 3 ) in Subunit IC. The downward increase in proportion of turbidites within Unit I likely corresponds to increasing sedimentation rates.

The onset of turbidite deposition at Site 827, which occurs at the base of Unit I, is abrupt and is characterized by the thickest and coarsest grain deposits. It is possible to constrain the timing of this onset using nannofossil-derived ages of 0.28 and $0.46 \mathrm{Ma}$ from Unit II. Extrapolation of a constant sedimentation rate $(15 \mathrm{~cm} / 1000 \mathrm{yrs})$ throughout Unit II suggests that turbidite deposition began at 110,000 yrs ago. However, if the uncertainties in nannofossil dating are taken into account, the first occurrence of turbidites could be as old as 160,000 yrs (Fig. 5). If this older age is adopted, much of Subunit IC would fall within isotopic Stage 6, with the initiation of intense turbidite deposition at about $82 \mathrm{mbsf}$ corresponding to the glacial maximum 150,000 years ago (Imbrie et al., 1984). The sedimentation rate curves in Figure 5 also suggest that the peak in turbidite frequency at $\sim 20 \mathrm{mbsf}$ in Subunit IA, Hole $827 \mathrm{~A}$, is between 18,000 and 38,000 yrs old. An age 
of $18,000 \mathrm{yrs}$ would suggest that this peak corresponds to the last glacial maximum (Imbrie et al., 1984) and is time correlative with the peak at $\sim 12 \mathrm{mbsf}$ in Hole 828A. We feel, therefore, that the evidence is suggestive of sea-level influence on turbidite deposition in the forearc, as shown by studies in other areas (e.g., Shanmugam and Moiola, 1982). Sea level is not, however, the sole factor influencing turbidite deposition at Site 827 , because cyclicity in depositional style is not evident at this site, which shows an overall decreasing trend in proportion of layers, maximum layer thickness and grain size with time from 90 to 0 mbsf (Figs. 3 and 4).

There are at least two hypotheses that could account for the upward decrease in proportion, thickness, and grain size of turbidites at Site 827. First, the source of sediment from turbidity currents could be becoming finer grained and less abundant during this time interval. Second, the direction of turbidity current flow to Site 827 may have changed as a result of tectonic processes. The first suggestion seems unlikely because Espiritu Santo Island has undergone significant uplift and emergence during the late Pleistocene, which would argue against a major fining of the sediment source. We, therefore, favor the second hypothesis, suggesting that progressive uplift of Site 827 and the upslope area to the east during middle to late Pleistocene time moved the site from a zone of intense turbidite deposition to a zone of diminished turbidite influence. Sediment deposited at Site 827 thus shifted from sandy/silty turbidites to silty/muddy turbidites, with the overall input modulated by sea-level changes. The initiation of turbidite deposition at the base of Unit I may have been a result of tectonic uplift, which created a depositional area for turbidites descending the inner trench slope as suggested by Collot, Greene, Stokking, et al. (1992).

\section{Site 829}

Site 829 is at the toe of the forearc slope, $2 \mathrm{~km}$ east of the trace of the subduction zone, at $2905 \mathrm{mbsf}$ (Fig. 1). Our post-cruise study of this site was restricted to Hole $829 \mathrm{~A}$, in which drilling penetrated $517.2 \mathrm{~m}$ of upper Pliocene to upper Pleistocene marine sediment overlying $73.1 \mathrm{~m}$ of volcanic breccia. Zones of structural deformation ranging in thickness from a few centimeters to $3 \mathrm{~m}$ are common in this sediment (see Meschede and Pelletier, this volume). The stratigraphic section was subdivided onboard ship into 16 lithostratigraphic units (Units I-XVI). Revised descriptions of these units based on post-cruise analyses are summarized below. The thicknesses of Units VIII-XII have been modified on the basis of Formation MicroScanner (FMS) data, which allowed us to position more accurately the boundaries between the carbonate and silt units in this interval of poor core recovery. The revised lithostratigraphy for Hole $829 \mathrm{~A}$ is shown in Figure 7.

Unit I (0-60.5 mbsf; Sections 134-829A-1R-1, $0 \mathrm{~cm}$, to -8R-1, 0 $\mathrm{cm})$ consists of lower or middle and upper Pleistocene (CN14 and $\mathrm{CN} 15)$ volcanic silt with a few thin sandy and ashy layers. The number of layers per section ranges from 0 to 4 (average $<1$ ), the mean thickness of the layers ranges from 1.1 to $2.2 \mathrm{~cm}$ (average $=1.4 \mathrm{~cm}$ ), and the maximum thickness of an individual layer is $2.2 \mathrm{~cm}$ (Fig. 3). Many of the layers are similar in composition to ash-rich layers at Site 828 , containing more glass than the sandy layers at Site 827. In the upper $10 \mathrm{~m}$ of Unit $\mathrm{I}$, the silt, which comprises the bulk of the unit, has a median grain size of $44 \mu \mathrm{m}$ and is moderately well sorted with a near symmetrical grain-size distribution (Fig. 4). The median grain size of the silt decreases to $20-23 \mu \mathrm{m}$ from 10 to $60 \mathrm{mbsf}$, and this sediment is poorly to moderately well sorted with a near symmetrical to coarsely skewed grain-size distribution (Fig. 4). The carbonate content of the silt increases from $9 \%-12 \%$ in the upper $40 \mathrm{~m}$ to $19 \%-37 \%$ in the lower $20 \mathrm{~m}$ of Unit I (Fig. 4 and Table 1).

Unit II (60.5-99.4 mbsf; Sections 134-829A-8R-1, $0 \mathrm{~cm}$, to -12R$1,40 \mathrm{~cm})$ consists of lower or upper Oligocene (CP17/18) and uppermost Oligocene or lower Miocene (CP19/CN1) foraminiferal chalk. Ten cm of brown clay occur at 99 mbsf (Interval 134-829-12R$1,0-10 \mathrm{~cm}$ ), about $30 \mathrm{~cm}$ above an abrupt contact between Oligocene chalk at the base of Unit II and a second interval of Pleistocene siltstone in Unit III. The brown clay consists of $65 \%$ smectite and $35 \%$ kaolinite.

Unit III (99.4-171.9 mbsf; Sections 134-829A-12R-1, $40 \mathrm{~cm}$, to $-19 \mathrm{R}-4,88 \mathrm{~cm}$ ) consists of lower or middle and upper Pleistocene (CN14 and CN15) volcanic silt and partially lithified siltstone; a biostratigraphic inversion at 148 mbsf places nannofossil Zone CN14 over a repeated section of CN15. Thin layers of volcanic sand and sandy silt, generally $<1 \mathrm{~cm}$ thick, occur with a frequency of $<1$ layer per section throughout Unit III. Granule- to pebble-sized clasts of pumice and upper and possibly lower Oligocene (CP17/18 and CP19) chalk occur throughout the unit. The median grain size of the silt is $16-18 \mu \mathrm{m}$ in the upper $40 \mathrm{~m}$ of the unit, but increases to coarse silt and sandy silt below about $140 \mathrm{mbsf}$. The carbonate content of the silt is variable, ranging from $5 \%$ to $30 \%$. The contact between Units III and IV is gradational.

Unit IV (171.9-205.2 mbsf; Sections 134-829A-19R-4, $88 \mathrm{~cm}$, to $-23 \mathrm{R}-1,0 \mathrm{~cm}$ ) consists of intensely deformed, lower or middle Pleistocene (CN14) siltstone-chalk breccia. The siltstone clasts are early or middle Pleistocene $(\mathrm{CN} 14)$ in age; the chalk clasts are middle Miocene (CN4) and Oligocene.

Unit V (205.2-378.4 mbsf; Sections 134-829A-23R-1, $0 \mathrm{~cm}$, to $-41 \mathrm{R}-1,0 \mathrm{~cm})$ consists of lower or middle Pleistocene (CN14) and lower Pleistocene or upper Pliocene (CN13) chalk breccia. The chalk clasts are late Oligocene or early Miocene (CP19/CN1) in age. The lower boundary of Unit V, which was placed at 311.4 mbsf onboard ship, has been shifted downward to include sediment now recognized as brecciated in the interval from 311.4 to 378.4 mbsf.

Unit VI (378.4-398.9 mbsf; Sections 134-829A-34R-1, $0 \mathrm{~cm}$, to $-43 \mathrm{R}-1,120 \mathrm{~cm}$ ) consists of unbrecciated lower or upper Oligocene (CP17/CP18) chalk and, in the lowermost $1.2 \mathrm{~m}$ of the unit (Sections 134-829A-43R-1, $0 \mathrm{~cm}$, to $-43 \mathrm{R}-1,120 \mathrm{~cm}$ ), upper Eocene (CP15, CP16/15) chalk with wavy laminations of brown, kaolinitic clay.

Unit VII (398.9-406 mbsf; Sections 134-829A-43R-1, $120 \mathrm{~cm}$, to $-43 R-C C)$ consists of matrix-supported volcanic breccia that is similar in composition to the volcanic breccia in Unit IV at Site 828 (see Coltorti et al., this volume). Brown clayey chalk is interbedded with the volcanic breccia in the upper $1.5 \mathrm{~m}$ of this unit. The clayey chalk is late Eocene (CP15) in age and contains kaolinite.

Unit VIII (406-413 mbsf; Sections 134-829A-44R-1, $0 \mathrm{~cm}$, to $-44 \mathrm{R}-1,40 \mathrm{~cm}$ ) consists of chalk with a poorly preserved early Pliocene(?) (CN11?) nannofossil assemblage. The FMS data suggest that the chalk unit represented by the $40 \mathrm{~cm}$ recovered in Core $134-829 \mathrm{~A}$ $44 \mathrm{R}$ is $7 \mathrm{~m}$ thick.

Unit IX (413-419.6 mbsf; Sections 134-829A-44R-1, $40 \mathrm{~cm}$, to $-45 R-C C)$, described Aboard ship as silty chalk, is redescribed as a partially lithified mixed sediment and silty chalk based on the total carbonate values, which range from $36 \%$ to $84 \% \mathrm{CaCO}_{3}$ (Table 1). The sediment is early or middle Pleistocene (CN14) in age.

Unit X (419.6-435 mbsf; Sections 134-829A-46R-1, $0 \mathrm{~cm}$, to $-47 \mathrm{R}-1,65 \mathrm{~cm}$ ) consists mainly of upper Oligocene or early Miocene (CP19/CN1) chalk with poorly preserved nannofossils; the sediment in Core 46R also contains rare Pleistocene (CN14) nannofossils, and may be slightly brecciated. In addition, $20 \mathrm{~cm}$ of upper Eocene (CP15) chalk with brown clay laminations occurs at the base of the unit.

Unit XI (435-442 mbsf; Sections 134-829A-47R-1, $65 \mathrm{~cm}$, to $-48 \mathrm{R}-\mathrm{CC}$ ), described onboard ship as silty chalk, is redescribed as a partially lithified mixed sediment based on the total carbonate values, which range from $37 \%$ to $54 \%$ (Table 1). This sediment is early or middle Pleistocene (CN14) in age. A $0.5-\mathrm{m}$ interval of chalk with poorly preserved lower Pliocene(?) (CN11?) nannofossils also occurs within the unit, possibly representing a boulder-sized clast.

Unit XII (442-463.2 mbsf; Sections 134-829A-49R-1, $0 \mathrm{~cm}$, to $-51 \mathrm{R}-3$, at $10 \mathrm{~cm}$ ) consists of lower Miocene or upper Oligocene 

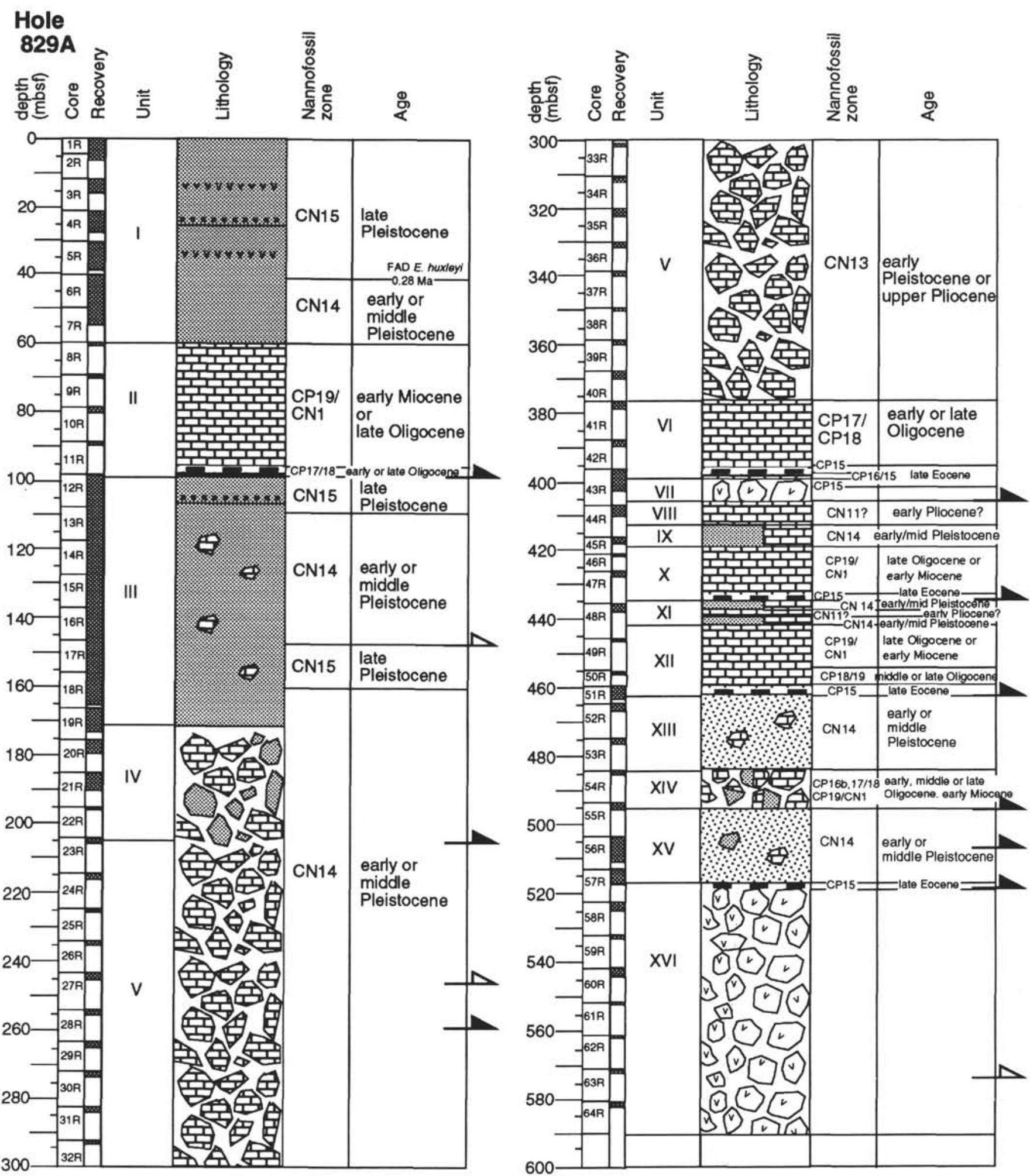

Figure 7. Revised lithostratigraphy for Site 829.

(CP19/CN1) and middle or upper Oligocene (CP18/19) chalk overlying upper Eocene (CP15) chalk with brown clay laminations; the mineralogy of the clay has not yet been analyzed.

Unit XIII (463.2-484.5 mbsf; Sections 134-829A-51R-3, 10 cm, to $-54 \mathrm{R}-1,0 \mathrm{~cm}$ ) consists of partially lithified, lower or middle Pleistocene (CN14) volcanic sandstone, sandy siltstone and conglom- erate. Plagioclase is abundant in the sandstone; pyroxenes, amphiboles, and opaque minerals such as magnetite are common. Chalk clasts, some with poorly preserved late Pliocene assemblages, occur throughout the unit.

Unit XIV (484.5-495.6 mbsf; Sections 134-829A-54R-1, $0 \mathrm{~cm}$, to $-55 \mathrm{R}-\mathrm{CC}, 15 \mathrm{~cm})$ consists of lower Oligocene (CP16b), middle 
or upper Oligocene (CP17/18), and upper Oligocene or lower Miocene $(\mathrm{CP} 19 / \mathrm{CN} 1)$ chalk and mixed sediment that is, at least in part, brecciated.

Unit XV (495.6-517.2 mbsf; Sections 134-829A-55R-CC, $15 \mathrm{~cm}$, to $-7 \mathrm{R}-3,72 \mathrm{~cm}$ ) consists of partially lithified, lower or middle Pleistocene $(\mathrm{CN} 14)$ volcanic sandstone and sandy siltstone. Clasts and contorted layers of lower Pliocene ( $\mathrm{CN10}$ ) and middle or upper Oligocene $(\mathrm{CP} 17 / 18)$ chalk and rounded siltstone clasts occur in the interval from 504.7 mbsf (Section 134-829A-56R-1, $87 \mathrm{~cm}$ ) to $506.7 \mathrm{mbsf}$ (Section 134-829A-56R-2, $137 \mathrm{~cm}$ ).

Unit XVI (517.2-590.3 mbsf; Sections 134-829A-57R-3, $72 \mathrm{~cm}$, to $64 \mathrm{R}-\mathrm{CC}, 27 \mathrm{~cm}$ ) consists of matrix- to grain-supported breccia composed mainly of fragments of volcanic rocks. In the upper part of the volcanic breccia, the matrix is laminated brown clay and chalk dated as late Eocene (CP15).

\section{Sedimentologic History}

The stratigraphic section at Site 829 records a story of tectonic accretion of ridge sediment and trench fill onto the forearc slope. Biostratigraphic inversions and structural deformation throughout the section are evidence that the section is sheared by numerous thrust faults, as shown in Figure 7.

Units I and II, which occur stratigraphically above the shallowest thrust fault at $99.4 \mathrm{mbsf}$, are interpreted as accreted ridge sediment with, more than likely, a drape of in-situ slope sediment. Because the compositions and textures of volcanic silt on the ridge (at Site 828) and forearc slope (Site 827) are similar, it is difficult to distinguish how much, if any, of the sediment in the upper part of Unit I at Site 829 represents in-situ deposition. The occurrence of glass-rich layers in the interval from 10 to $35 \mathrm{mbsf}$ may be an indication that the section below 10 mbsf was accreted from the ridge, as similar layers occur at Site 828 but not at Site 827; however, ashy layers could have been obliterated by erosion or reworking at Site 827 and yet still be present elsewhere on the slope. The chalk that comprises Unit II is younger (CP17/18 and CP19/CN1) than the ooze at Site 828 (CP15 to CP17/18), but lower Miocene to lower Oligocene carbonate sediment was dredged from Bougainville Guyot by Collot et al. (1992). The brown clay at the base of Unit II, Site 829, which occurs above Oligocene (CP17/18) chalk, is also younger than the brown clay at Site 828 , which occurs within upper Eocene ooze (CP15), and is compositionally distinct, containing smectite and kaolinite (the clay at Site 828 contained only kaolinite). Both smectite and kaolinite can form authigenically or can occur as detrital minerals (Blatt et al., 1980). Smectite typically forms from the alteration of volcanic ash and tuff; kaolinite is commonly derived from altered feldspars. Both the smectite and kaolinite in Unit II at Site 829 could have been derived by weathering an exposed volcanic ridge.

Units III to VII are thought to represent accreted ridge material overlain by trench fill deposits. Although the occurrence of at least four thrust faults within this interval (see Fig. 7) suggests that these units may not represent a single depositional sequence, the ages and lithologies show trends that could be original. Units VI and VII, which consist of middle or upper Oligocene chalk and upper Eocene chalk with laminations of kaolinitic clay overlying volcanic breccia, are interpreted as accreted ridge sediment because they are equivalent in age and lithology to sediment at Site 828. Units III, IV, and V, which grade upward from upper Pliocene to lower or middle Pleistocene chalk breccia (Unit V), to lower or middle Pleistocene chalk-siltstone breccia (Unit IV) and early or middle to upper Pleistocene siltstone with occasional chalk clasts (Unit III), are interpreted as accreted trench fill deposits. The chalk clasts in these units, which range in age from middle Oligocene to middle Miocene, could have been eroded from the downgoing plate or from previously accreted chalk on the forearc slope. The middle or late Oligocene clasts (CP17/18) are age-equivalent to ooze at Site 828; however, late Oligocene to middle Miocene (CP19/CN1 and CN4) ooze was not recovered at Site 828.
Like Units III to VII, Units VIII to XII could also be slivers of ridge rocks overlain by trench fill. Units X and XII, which consist mainly of upper Eocene to upper Oligocene or lower Miocene chalk, appear to be pieces of ridge that were scraped off above volcanic basement at the brown clay horizon. Units VIII, IX, and XI could be trench fill deposits consisting of boulders or slabs of Pliocene chalk in a matrix of lower or middle Pleistocene mixed sediment.

Units XIII to XV are interpreted as accreted trench fill composed of material derived from both the arc and the downgoing plate. Amphiboles in the volcanic sand in Units XIII and XV suggest the erosion of the Western Belt islands, probably Espiritu Santo. Pliocene, Oligocene, and possibly lower Miocene chalk clasts in Units IV, XIII, and XV were probably derived from the downgoing plate or from chalk previously accreted to the forearc slope. Unit XVI is interpreted as accreted ridge material because it is lithologically similar to volcanic breccia at Site 828 (Coltorti et al., this volume).

If the thrust sheets at Site 829 were emplaced in stratigraphic order, one under the other, the first accreted thrust sheet would be the one highest in the section (i.e., the sheet represented by Units I and II). Examination of the nannofossil age data at Site 829 suggests, however, that the stratigraphic section may not be in order, and that more recently accreted sediment may overlie older accreted sediment. The age of emplacement of the uppermost thrust sheet (Units I and II) is uncertain because we do not know how much of the volcanic silt in Unit $\mathrm{I}$ is accreted and how much was deposited on the forearc slope. However, the presence of upper Pleistocene (CN15) silt in the upper part of Unit III, in the second thrust sheet, suggests that accretion of Units I to III occurred within the past 280,000 yrs. On the other hand, the youngest sediment in Units IV to XV is early to middle Pleistocene (CN14) in age, which suggests that accretion of these units occurred within the past 1.58 m.y. The lack of Upper Pleistocene (CN15) sediment in Units IV to XV suggests that accretion of these units predated accretion of Units I to III. A possible explanation for the stratigraphy is that sediment in Units I to III was thrust over previously accreted sediment in Units IV to XV.

\section{DISCUSSION AND CONCLUSIONS}

The stratigraphy of Sites 828,827 , and 829 provide an opportunity to observe the effects of the subduction of the NDR on in-situ sedimentary processes and tectonic accretion in the forearc area of the New Hebrides Island Arc. One of the most pronounced consequences of the NDR subduction is the dramatic uplift of the forearc area to form the emergent islands of Espiritu Santo and Malakula (Fig. 1). The uplift has been especially significant during the Pleistocene and has provided an abundant supply of epiclastic sediment that is preferentially distributed to the west by the prevailing ocean currents. This influx of volcaniclastic material has dominated the sedimentation of areas on and at the base of the inner trench slope, and to some extent, parts of the eastward-moving NDR. The sediment has been delivered to the deep water environment by numerous turbidity currents, most likely generated either by earthquakes or strong storms.

Site 828 records an abrupt change from dominantly pelagic sedimentation to strongly hemipelagic sedimentation with abundant turbidites. This shift represents translation of the site by relative plate movement $(13 \mathrm{~cm} / \mathrm{yr})$ into the zone of turbidite influence. However, the abundance of turbidites does not simply increase progressively as the ridge moved closer to the arc; there is a distinct peak at about 12 mbsf. The age of this peak can be correlated with the last glacial maximum, 18,000 yrs ago, suggesting that sea level changes may have also contributed to variations in the frequency of turbidites, as has been suggested by several other workers (Droxler and Schlager, 1985; Shanmugam and Moiola, 1982).

Turbidite deposits are also abundant at Site 827 and to a lesser extent at Site 829 . These sites have probably not suffered extensive lateral translation, but may have undergone significant vertical movement 
associated with the subduction of the NDR. Abrupt initiation of turbidite deposition at Site 827 was preceded by accumulation of a thick section of hemipelagic sediment. An earlier suggestion that turbidite deposition at Site 827 was initiated by the formation of a trapping basin (Collot, Greene, Stokking, et al., 1992) seems reasonable, but does not fully explain the temporal changes in turbidite frequency, grain size, or accumulation rate observed at this site. Our data suggest that turbidite deposition at Site 827 was modulated by sea level changes and by continued uplift of the site, which, after first creating the basin, progressively isolated it from the direct influence of coarse and thick turbidity currents. The sedimentary record at Site 827 thus bears some similarity to the uplifted trench slope sedimentary sequence exposed on Nias Island off the coast of Sumatra. The Nias sequence shows an upward thickening and coarsening trend that has been interpreted as the result of uplift of an isolated basin into the influence of a submarine canyon system that supplied coarse, basinfilling turbidites (Moore et al., 1980). Unlike Nias Island, however, uplift at Site 827 continued to a point where the area once again became relatively isolated from coarse grain turbidites. The rapid passage of Site 827 through contrasting sedimentary environments can be attributed to tectonic processes associated with NDR subduction.

The primary consequences of the NDR subduction for in-situ trench-slope sedimentation has thus been to: (1) initiate a new source of epiclastic sediment through uplift and erosion of Espiritu Santo Island; and (2) cause vertical and horizontal translation of depositional sites, which are recorded as variations in the lithology, frequency, grain size, and volume of turbidite deposits. In addition, sea level fluctuations associated with glacial-interglacial cycles have acted independently to modulate the influx of sediment to the trench slope area.

Previous studies of accretion at convergent margins (e.g., Karig, 1974; Karig and Sharman, 1975; Moore, 1975) have noted that pelagic sediment tends to be subducted and terrigenous trench deposits accreted at the toes of accretionary prisms. The authors of these studies suggested that changes in mechanical properties of sediment in subduction zones lead to detachment of strong trench deposits from pelagic sediment along relatively weak hemipelagic deposits. Vrolijk (1990) suggested that décollements in subduction zones tended to occur in smectite-rich horizons, which are common in hemipelagic sediment.

Accretion associated with the collision of the NDR with the New Hebrides Island Arc does not conform to previous models. Both pelagic sediment and trench deposits have been accreted onto the inner trench slope east of the collision zone, and the décollement appears to have formed along kaolinite-rich, rather than smectite-rich, horizons.

Calcareous pelagic sediment accreted from the NDR onto the forearc slope at Site 829 is similar in age to sediment at Site 828: late Eocene (CP15), early or late Oligocene (CP17/18), and early Pliocene (CN11) assemblages are found at both locations. However, late Oligocene or early Miocene (CP19/CN1) pelagic sediment occurs at Site 829 , but not at Site 828 , suggesting that the 25 m.y. unconformity between Oligocene and Pliocene sediment observed at Site 828 may vary in duration along the ridge. In addition, dewatering of pelagic sediment caused by compressional stress during accretion is suggested by measurements of water content, which are $50 \%-70 \%$ in ooze at Site 828 and $10 \%-25 \%$ in chalk at Site 829 (Collot, Greene, Stokking, et al., 1992; Leonard and Ask, this volume).

Accreted sediment on the forearc slope that was originally deposited in the trench occur as silt, sand and conglomerate containing clasts eroded from both the arc and ridge sides of the trench. This trench fill sediment is early, middle, and late Pleistocene (CN13, CN14, CN15) in age. Our interpretation that this sediment was originally deposited within the trench, rather than as basin fill on the trench slope, is based on the proximity of the site to the trench and the highly deformed nature of the sequence (see Underwood and Bachman, 1982). Chalk clasts within the trench fill sediment, which are thought to be derived from the ridge, are generally similar in age to calcareous ooze at Site 828 (early or upper Oligocene, CP17/18, and lower Pliocene, CN11, faunas occur in both); however, some of the chalk clasts also contain late Oligocene or early Miocene (CP19/CN1) and middle Miocene (CN4) assemblages. This again suggests that the pre-Pliocene unconformity on the ridge is variable in duration.

The ages of accreted sediment on the forearc slope at Sites 827 and 829 can be used to constrain the timing of accretion. As discussed previously, the ages suggest that there has been accretion at both sites within the past $1.58 \mathrm{~m}$.y., with at least some additional accretion at Site 829 during the past 280,000 yrs.

Offscraping of sediment from the subducting ridge seems to have occurred preferentially at kaolinite-rich horizons at the base of the pelagic sequence above the volcanic basement. Similarities of age and mineralogic composition of clay-rich horizons on the ridge at Site 828 and clays associated with thrust faults at Site 829 suggest that the clays provided a preferential zone of separation along which ridge rocks were detached from the downgoing plate and accreted onto the trench slope. The clay at Site 828 is upper Eocene (CP15) in age and the only clay mineral detected in XRD analysis was kaolinite. The clay associated with all but one of the thrust faults at Site 829 is also upper Eocene (CP15 or CP16/15) and kaolinite was the only clay mineral detected in two of three samples analyzed with XRD. Smectite, which has been associated with other décollement zones (Vrolijk, 1990), was found in only one sample at the base of Unit II in Hole 829A; this clay is Oligocene (CP17/18) in age and contains $65 \%$ kaolinite, in addition to $35 \%$ smectite. Further study of clay mineralogy and physical properties are needed to determine the potential role of kaolinite or other clays in the formation of the décollement in the collision zone between the NDR and the New Hebrides Island Arc.

\section{SUMMARY}

Revisions to the descriptions and interpretations of the lithostratigraphic units defined by Collot, Greene, Stokking, et al. (1992), based on post-cruise analysis of sediment from Sites 828,827 , and 829 can be summarized as follows:

\section{Site 828}

1. The upper $50 \mathrm{~m}$ section of Hole $828 \mathrm{~A}$, formerly dated as middle Pleistocene, is now assigned a late Pleistocene age.

2. Sediment in the interval from 58.7 to $61.9 \mathrm{mbsf}$ (Sections $134-828 \mathrm{~A}-7 \mathrm{H}-5,83 \mathrm{~cm}$, to $8 \mathrm{H}-1,48 \mathrm{~cm}$ ), formerly described as foraminiferal ooze and placed in Subunit IIA, is redescribed as mixed sediment and included within Unit I because of the general increase in $\mathrm{CaCO}_{3}$ throughout the lower $10 \mathrm{~m}$ of Unit $\mathrm{I}$.

3. Sediment in Unit III is redescribed as firm ooze, rather than chalk, because the sediment is not brittle and deforms with finger pressure.

4. Brown clay laminations have been identified in upper Eocene chalk at the base of Unit III in Hole 828B.

5. Pleistocene sedimentation at Site 828 is interpreted to reflect movement of the NDR toward the New Hebrides Island Arc, uplift of Espiritu Santo Island and sea level changes.

\section{Site 827}

1. The boundaries of most of the nannofossil zones have been shifted and Zones $\mathrm{CN} 12$ and $\mathrm{CN} 14$ have been subdivided.

2. The base of Subunit IC is placed at $88.4 \mathrm{mbsf}$, rather than at 86 mbsf as defined aboard ship, to include sandy layers that occur below Section 827A-12H-2, $90 \mathrm{~cm}$.

3. Sediment in Unit IV and at the base of Unit III is interpreted as accreted because a biostratigraphic inversion indicative of a thrust fault has been identified at the base of Unit III. Accretion has occurred within the past $1.58 \mathrm{Ma}$.

4. Pleistocene deposition at Site 827 is thought to reflect progressive uplift of the site and changes in sea level. The onset of turbidite 
deposition at the base of Unit I is attributed to a combination of the low sea level stand representing the $150,000 \mathrm{yr}$ glacial maximum and tectonic adjustments, which made the site suitable for turbidite deposition.

\section{Site 829}

1. The boundaries of most of the nannofossil zones have been modified and additional zones have been added.

2. A biostratigraphic inversion has been identified in Unit III, placing sediment in nannofossil Zone $\mathrm{CN} 14$ over those in Zone $\mathrm{CN} 15$.

3. The boundary between Units V and VI has been shifted from 311.3 to 378.4 mbsf to include sediment now recognized as brecciated in this interval.

4. The thicknesses of Units VIII-XII have been modified based on FMS data.

5. Sediment in Units IX and XI, formerly described as silty chalk, is redescribed as mixed sediment with minor silty chalk based on the gasometric analysis of the carbonate contents.

6. Units III, XIII, XV, and possibly also Units IX and XI, which were formerly interpreted as pieces of accreted ridge, are reinterpreted as accreted trench fill deposits.

7. The brown clay associated with several of the thrust fault zones, which was previously thought to be diagenetic, is reinterpreted as a preferred zone of separation along which ridge rocks are detached from the downgoing plate and accreted to the forearc slope because this clay is similar in age and composition to brown clay at the base of the pelagic sequence at Site 828 .

\section{ACKNOWLEDGMENTS}

This research was supported by JOI-USSAC grants to R.P. Reid and T.S. Staerker. We thank the personnel of the ODP Core Repository, Texas A\&M University, for help with core sampling. We are also grateful to the Texas Veterinary Medical Center, Texas A\&M University, and in particular to Joni Elslander, for assistance with X-radiography. Clay mineralogy was analyzed by Michael Lutz at the Rosenstiel School of Marine and Atmospheric Science (RSMAS), University of Miami.

\section{REFERENCES*}

Biscayne, P.E., 1965. Mineralogy and sedimentation of recent deep-sea clays in the Atlantic Ocean and adjacent seas and oceans. Geol. Soc. Am. Bull., $76: 803-832$.

Blatt, H., Middleton, G.V., and Murray, R., 1980. Origin of Sedimentary Rocks (2nd ed.): Englewood Cliffs, NJ (Prentice-Hall).

Collot, J.-Y., Daniel, J., and Burne, R.V., 1985. Recent tectonics associated with the subduction/collision of the d'Entrecasteaux zone in the central New Hebrides. Tectonophysics, 112:325-356.

Collot, J.-Y., and Fisher, M.A., 1992. The D'Entrecasteaux Zone-New Hebrides Island Arc collision zone: an overview. In Collot, J.-Y., Greene, H.G., Stokking, L.B., et al., Proc. ODP, Init. Repts., 134: College Station, TX (Ocean Drilling Program), 19-31.

Collot, J.-Y., Greene, H.G., Stokking, L.B., et al., 1992. Proc. ODP, Init. Repts., 134: College Station, TX (Ocean Drilling Program),
Collot, J.-Y, Lallemand, S., Pelletier, B., Eissen, J.-P, Glaçon, G., Fisher, M.A., Greene, H.G., Boulin, J., Daniel, J., and Monzier, M., 1992. Geology of the d'Entrecastaux-New Hebrides island arc collision: results from a deep-sea submersible survey. Tectonophysics, 212:213-241.

Dolan, J., Beck, C., and Ogawa, Y., 1989. Upslope deposition of extremely distal turbidites: an example from the Tiburon Rise, west-central Atlantic. Geology, 17:990-994.

Droxler, A.W., and Schlager, W., 1985. Glacial versus interglacial sedimentation rates and turbidite frequency in the Bahamas. Geology, 13:799-802.

Imbrie, J., Hays, J.D., Martinson, D.G., McIntyre, A., Mix, A.C., Morley, J.J., Pisias, N.G., Prell, W.L., and Shackleton, N.J., 1984. The orbital theory of Pleistocene climate: support from a revised chronology of the marine delta $\delta^{18} \mathrm{O}$ record. In Berger, A., Imbrie, J., Hays, J., Kukla, G., and Saltzman, B. (Eds.), Milankovitch and Climate (Pt. 1): Dordrecht (D. Reidel), 269-305.

Jones, G.A., and Kaiteris, P., 1983. A vacuum-gasometric technique for rapid and precise analysis of calcium carbonate in sediments and soils. $J$. Sediment. Petrol., 53:655-660.

Karig, D.E., 1974. Evolution of arc systems in the western Pacific. Annu. Rev. Earth Planet. Sci., 2:51-75.

Karig, D.E., and Sharman, G.F., III, 1975. Subduction and accretion in trenches. Geol. Soc. Am. Bull., 86:377-389.

Macfarlane, A., Carney, J.N., Crawford, A.J., and Greene, H.G., 1988. Vanuatu-a review of the onshore geology. In Greene, H.G., and Wong, F.L. (Eds.), Geology and Offshore Resources of Pacific Island Arcs-Vanuatu Region. Circum-Pac. Counc. Energy and Miner. Resour., Earth Sci. Ser., 8:45-91.

Maillet, P., Monzier, M., Selo, M., and Storzer, D., 1983. The d'Entrecasteaux zone (southwest $\mathrm{Pacific}$ ). A petrological and geochronological reappraisal. Mar. Geol., 53:179-197.

Moore, D.M., and Reynolds, R.C., Jr., 1989. X-ray Diffraction and the Identification and Analysis of Clay Minerals: Oxford (Oxford Univ. Press).

Moore, G.F., Billman, H.G., Hehanussa, P.E., and Karig, D.E., 1980. Sedimentology and paleobathymetry of Neogene trench-slope deposits, Nias Island, Indonesia. J. Geol., 88:161-180.

Moore, J.C., 1975. Selective subduction. Geology, 3:530-532.

Muck, M., and Underwood, M., 1990. Upslope flow of turbidity currents: a comparison among field observations, theory, and laboratory models. Geology, 18:54-57.

Shanmugam, R., and Moiola, R., 1982. Eustatic control of turbidites and winnowed turbidites. Geology, 10:231-235.

Taylor, F.W., 1992. Quaternary vertical tectonics of the central New Hebrides Island Arc. In Collot, J.-Y., Greene, H.G., Stokking, L.B., et al., Proc. ODP, Init. Repts., 134: College Station, TX (Ocean Drilling Program), 33-42.

Underwood, M., and Bachman, S., 1982. Sedimentary facies associations within subduction complexes. In Leggett, J.K. (Ed.), Trench-Forearc Geology. Geol. Soc. Spec. Publ. London, 10:537-550.

Vrolijk, P., 1990. On the mechanical role of smectite in subduction zones. Geology, 18:703-707.

\footnotetext{
- Abbreviations for names of organizations and publications in ODP reference lists follow the style given in Chemical Abstracts Service Source Index (published by American Chemical Society).
}

Date of initial receipt: 9 April 1992

Date of acceptance: 22 March 1993

Ms 134SR-005 